\title{
Dielectric Constant and
}

Electrical Resistivity of

Natural-State Cores

GEOLOGICAL SURVEY BULLETIN 1052 - H

This report concerns work done on behalf of the U.S. Atomic Energy Commission and is published with the permission of the Commission 


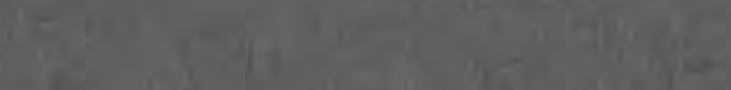

(1)

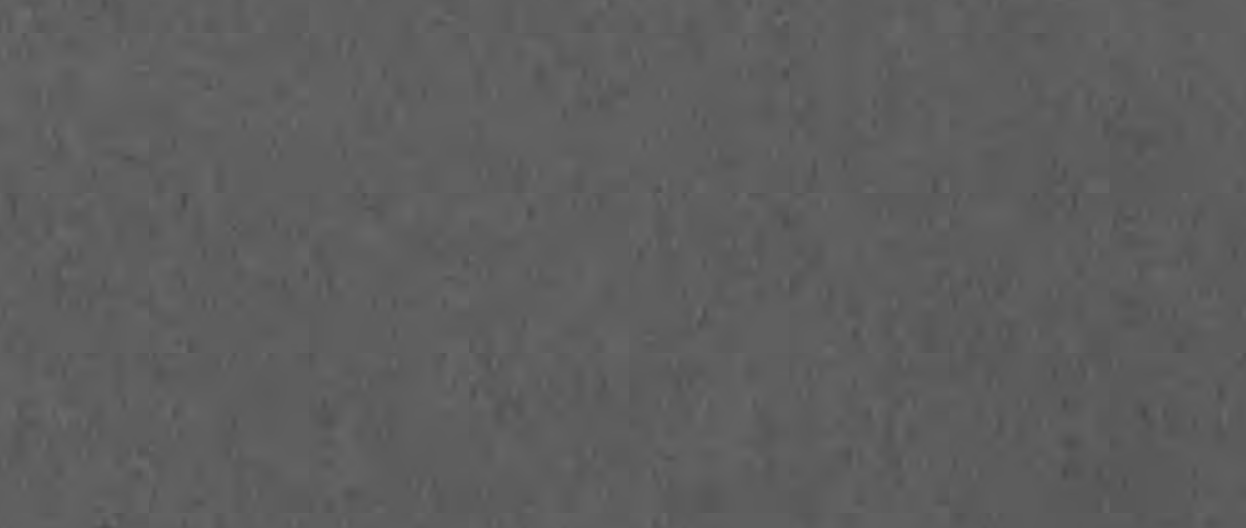

(4) $\frac{14}{43}$

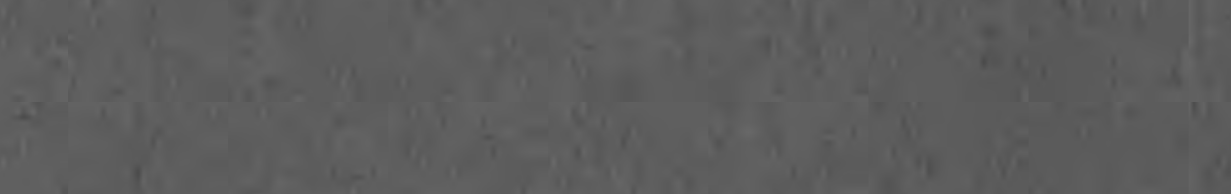

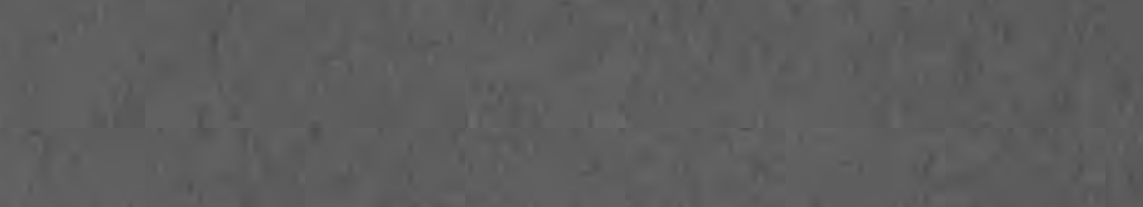

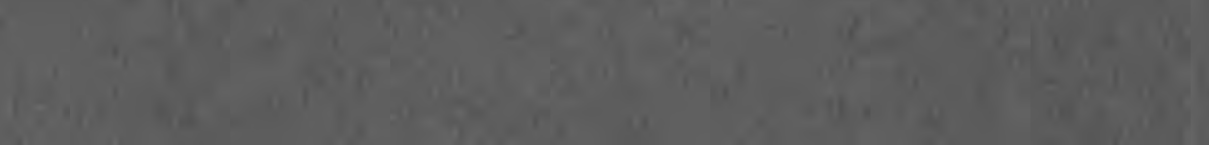

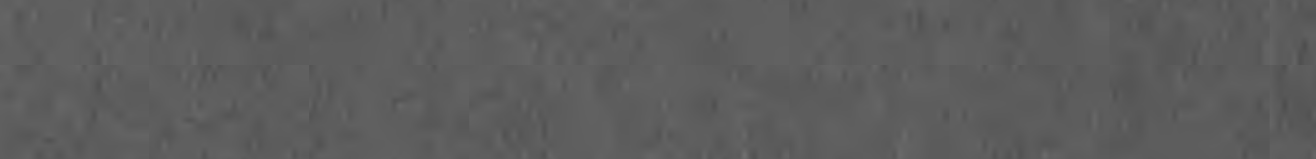

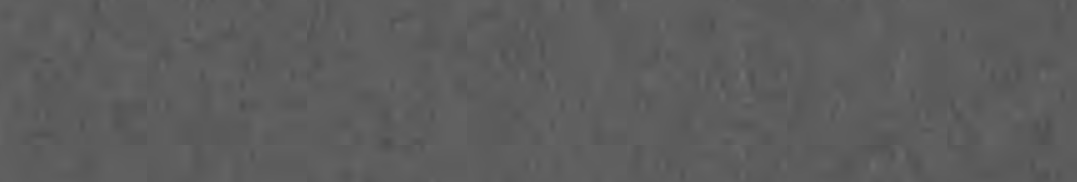

8

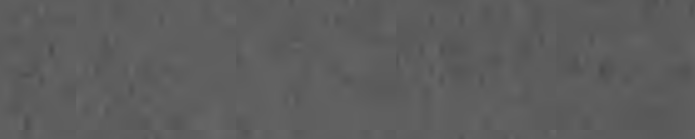

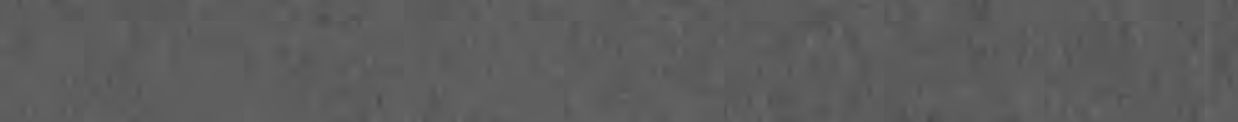
$+5$

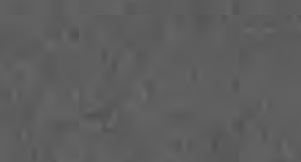

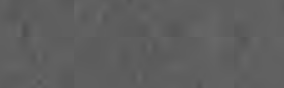

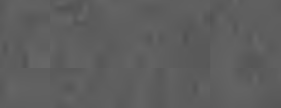

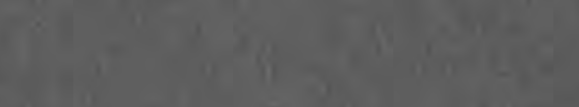

(1) yil

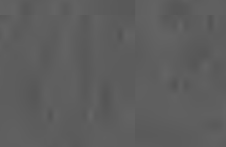

1

1. $=$ $t^{2}$

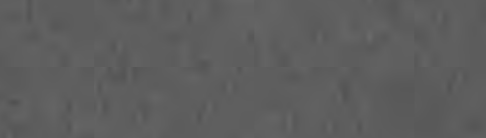

$y=$

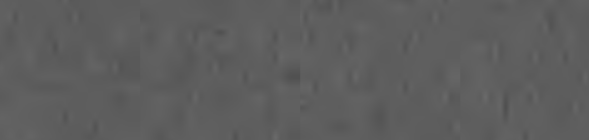

( 


\title{
Dielectric Constant and
} Electrical Resistivity of Natural-State Cores

\author{
By G. V. KELLER and P. H. LICASTRO
}

EXPERIMENTAL AND THEORETICAL GEOPHYSICS

GEOLOGICAL S U R V E Y B U L L E T I N $1052-\mathrm{H}$

This report concerns work done on behalf of the U.S. Atomic Energy Commission and is published with the permission of the Commission

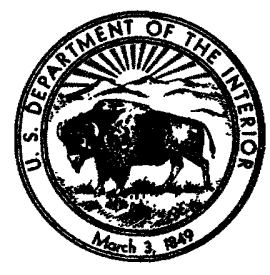


UNITED STATES DEPARTMENT OF THE INTERIOR

FRED A. SEATON, Secretary

\section{GEOLOGICAL SURVEY}

Thomas B. Nolan, Director 


\section{CONTENTS}

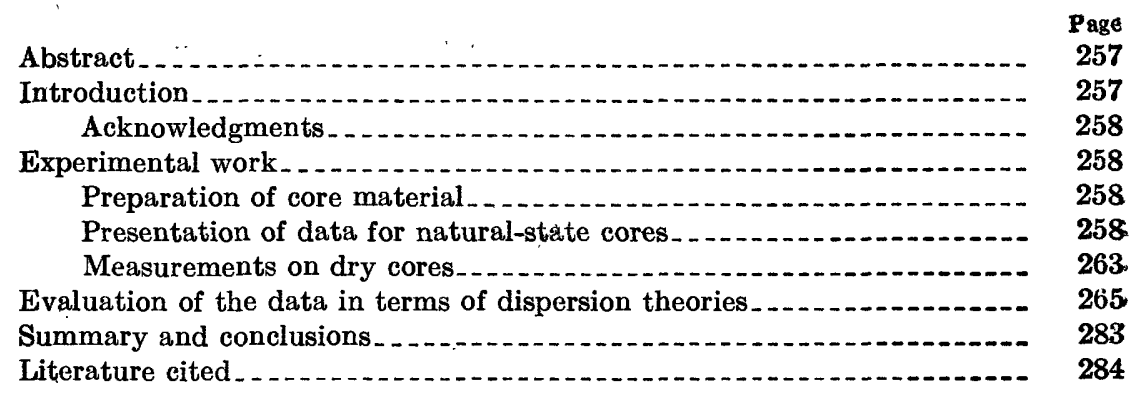

\section{ILLUSTRATIONS}

FiaURE 68. Dielectric constants of natural-state Morrison cores

69. Resistivity of natural-state Morrison cores

70. Loss angles measured on natural-state Morrison cores.....-

71. Phase angle versus water content at a frequency of $10 \mathrm{kc}$

72. Dielectric constants of dried Morrison cores measured in vacuum

73. Equivalent circuit for electrode polarization

74. Dispersion curves showing effect of electrode-polarization capacity decaying as an inverse power of the frequency ....

75. Static dielectric constant versus water content .............

76. Distribution times as a function of the distribution parameter $r_{-}$

77. Wagner's equation for dispersion of the real dielectric constant in a substance with a normal distribution of relaxation times

78. Equation for dispersion of the imaginary dielectric constant in a substance with a normal distribution of relaxation times.

79. Dielectric ratio as a function of distribution parameter and frequency

80. Example of the graphical determination of Wagner's parameters $b$ and $z_{0}$

81. Theoretical dielectric constant as a function of distribution parameter and frequency

82. Graphical determination of the maximum dielectric constant and the parameter $k$ of Wagner's equation

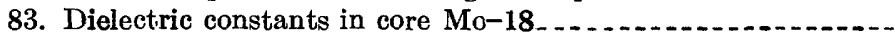


Fraure 84. Equivalent circuit for a dielectric material having more than

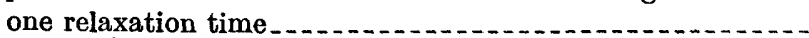

85. Dielectric constant for a laminated mixture of quartz and water

86. Idealized pore structure

87. Tracing made from a photomicrograph of a stained thin section, showing the pore structure of a typical sandstone in

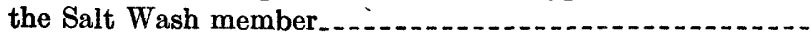

88. Number of immobile cations in a typical sandstone of the Salt Wash member

\section{TABLES}

TABLE 1. Characteristics of the dielectric dispersion of natural-state

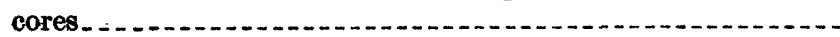

2. Evaluation of dielectric constant under certain hypothetical

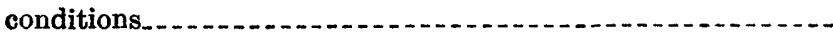




\title{
EXPERIMENTAL AND THEORETICAL GEOPHYSICS
}

\section{DIELECTRIC CONSTANT"AND ELEGTRIGAL RESISTIVITY OF NATURAL-STATE_CORPS}

\author{
By G. V. Keller and P. H. Licastro
}

\begin{abstract}
As part of a program to obtain basic data on the physical properties of the rock in and near uranium-mineralized zones, measurements of electrical resistivity and dielectric constant were made on parts of 27 cores from the Morrison formation in the Colorado Plateau uranium province. For frequencies between 50 cycles and 30 megacycles per second, resistivity ranged from $10^{2}$ to $10^{12} \mathrm{ohm}$-centimeters, and the dielectric constant ranged from 4.0 to more than $10^{6}$. The water content of the cores seemed to be the controlling factor: the high resistivities were associated with low water content and the high dielectric constants with high water content.
\end{abstract}

\section{INTRODUCTION}

The electrical properties of the Morrison formation in the Uravan mineral belt of the Colorado Plateau have been measured in place by electric logging and in the laboratory on cores with restored water saturation (Keller, 1959). The resistivity of different members of the Morrison formation in drill holes ranged from 8 to $2,000 \mathrm{ohm}$ meters. The resistivity of the sandstone members of the Morrison was found to be highest in areas that were considered most favorable for the occurrence of ore, probably because of the lower water saturation or lower salinity of the water in the favorable areas.

During 1952 a program of natural-state coring was begun by the U.S. Geological Survey in the Long Park area (Manger, Gates, and Cadigan, 1954) to obtain basic data on physical properties of rocks in and near uranium-mineralized zones. Drilling was done using air and oil-base drilling mud so that the water content of the cores could be preserved as nearly as possible in its original condition. These cores offered an opportunity to obtain truly representative values of the resistivity.

Parts of 27 cores of sandstones and mudstones of the Morrison formation, taken from 2 drill holes in the Long Park area, were used in 
this study. Both dielectric constant and electrical resistivity were measured from frequencies of 50 cycles to 30 megacycles per second.

\section{ACKNOWLEDGMENTS}

The work herein reported was done in part under the program carried on by the Geological Survey on behalf of the Division of Raw Materials, U.S. Atomic Energy Commission. Some of the laboratory work was carried out at the Pennsylvania State University, where equipment and laboratory facilities were made available by the University.

\section{EXPERIMENTAL WORK}

\section{PRIPARATION OF CORE MATERIAL}

For measurements of dielectric constant and electrical resistivity, a thin wafer was cut from each of the cores. The plane faces of these wafers were coated with conductive silver paint to form a pair of electrodes. The capacity and resistance of each piece of core were determined by using the core as one arm of a bridge circuit. A second arm contained a capacitor and resistor which were varied to match the capacity and resistance of the core. The bridge was operated at discrete frequencies from 50 cycles per second to 30 megacycles per second.

The core holder consisted of a brass plate placed in contact with the bottom of a core and a point electrode on a micrometer adjustment in contact with the top. Use of a conical-point electrode, which has practically no capacitance of its own, and wafer-shaped cores eliminated the necessity. for Kelvin shielding of the cores and reduced considerably the complexity of the bridge adjustment.

\section{PRESENTATION OF DATA FOR NATURAL-STATE CORES}

The dielectric constants measured on the 27 natural-state cores are presented graphically in figure 68 . The data are presented as dielectric ratios: that is, the capacity of a core divided by the capacity that an air condenser of the same dimensions would have. In order to obtain dielectric constants in cgs units from figure 68 , it is necessary to multiply the ratios by the factor $8.85 \times 10^{-4}$ farads per centimeter, the dielectric constant of air.

The values of dielectric constant are strongly dispersed, ranging from 4 to more than $10^{6}$ at the low frequency end of the spectrum. At frequencies above 10 megacycles per second, the dielectric constants are all less than 80 , as might be expected. In cores with very low water saturations, such as Nos. 10 and 12, there is almost no variation of dielectric constant with frequency. 


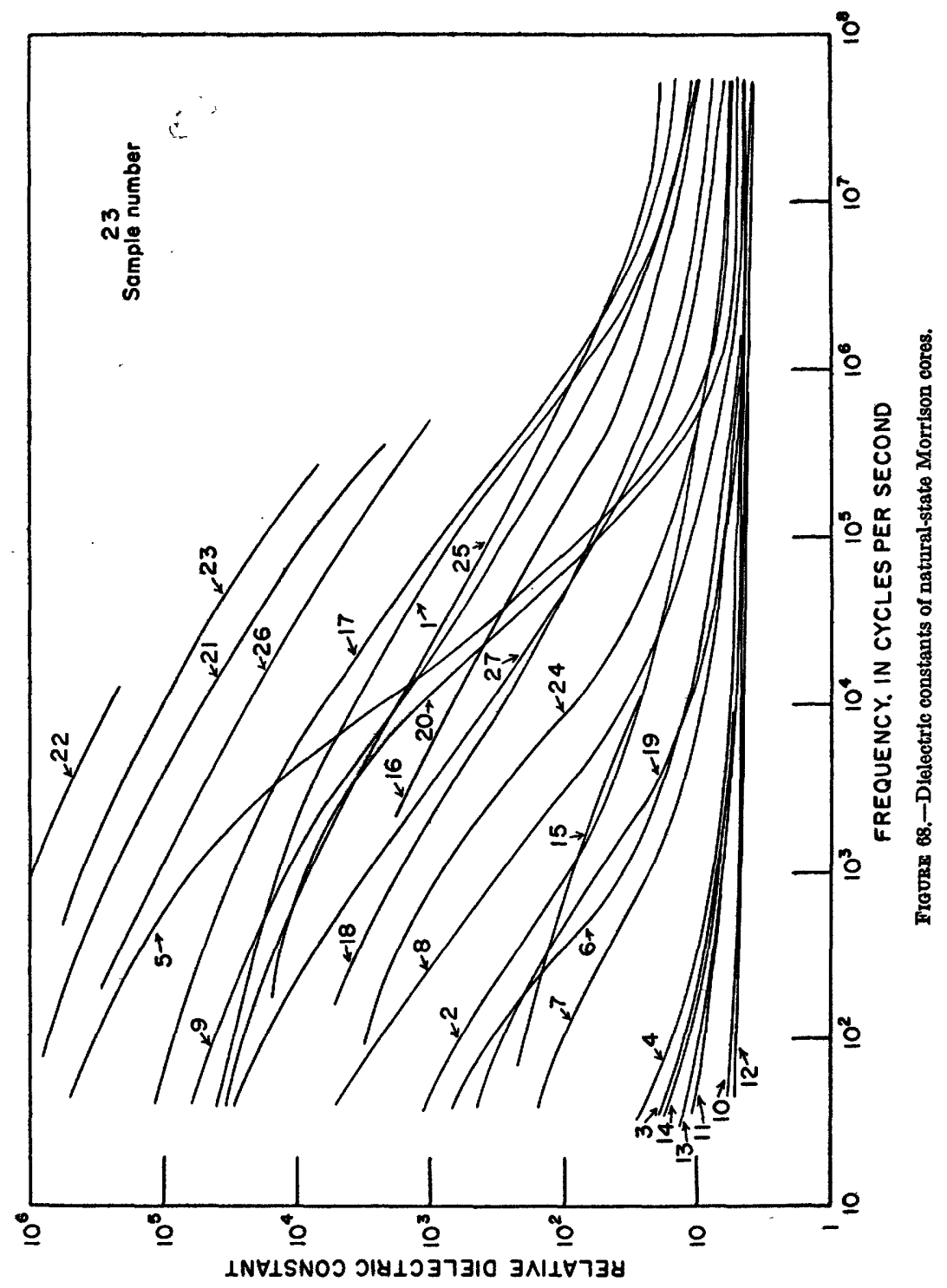




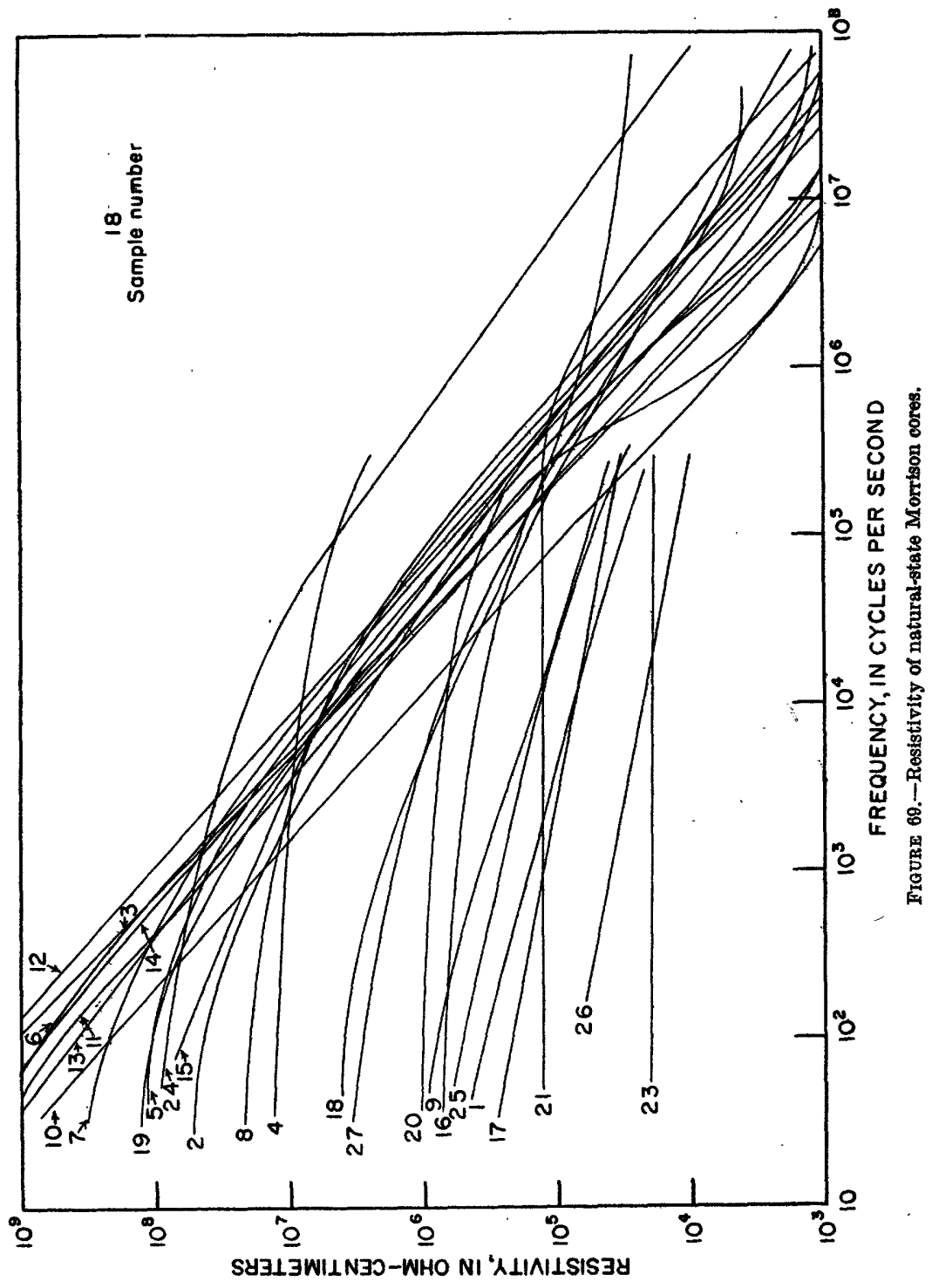


The results of the resistivity measurements on these same cores are presented in figure 69 in cgs units, ohm-centimeters. In the dry cores (see, for example, Nos. 10 and 12) there is a linear inverse relation between resistivity and frequency over practically the entire range of frequencies studied. On the other hand, in the wet cores such as Nos. 21 and 23 there is no variation of resistivity with frequency.

The dispersion of resistivity and the anomalous behavior of dielectric constant raise a question as to which is the more important in controlling current flow. When a voltage is impressed on a material, the current that flows can be considered as consisting of two parts: one in phase with the applied voltage, resulting in ohmic conductivity; and another in quadrature with the applied voltage, termed the displacement current. The ratio of these two currents is the tangent of the phase angle between the applied voltage and the resultant current and is a measure of the relative importance of resistivity and dielectric constant in determining the response of a material in an applied electric field. The ratio of ohmic current to displacement current was determined for each of the 27 cores by

$$
\tan \delta=\frac{1}{\omega \in \epsilon_{0} \rho}
$$

where

$\delta=$ the phase angle

$\epsilon=$ the relative dielectric constant of a core

$\epsilon_{0}=$ the dielectric constant of air

$\omega=$ the frequency in radians per second

$\rho=$ the electrical resistivity

The phase angles are shown graphically in figure 70.

Conduction in the cores studied ranges from predominantly ohmic $(\tan \delta>1)$ to predominantly displacement conduction $(\tan \delta>1)$. The cores with the highest dielectric constant are those in which conduction is almost entirely ohmic; and in those with very low dielectric constant, conduction is almost entirely by displacement currents.

The transition from displacement conduction at low water content to ohmic conduction at high water content is relatively sharp. This is shown in figure 71 by the phase-angle data at 10 kilocycles per second (kc). At this frequency the transition occurs at a water content of 3 percent. At lower frequencies the transition takes place at lower water contents; but even at frequencies as low as 50 cycles per second, the quadrature component of conduction was important in some of the sandstone cores studied. 


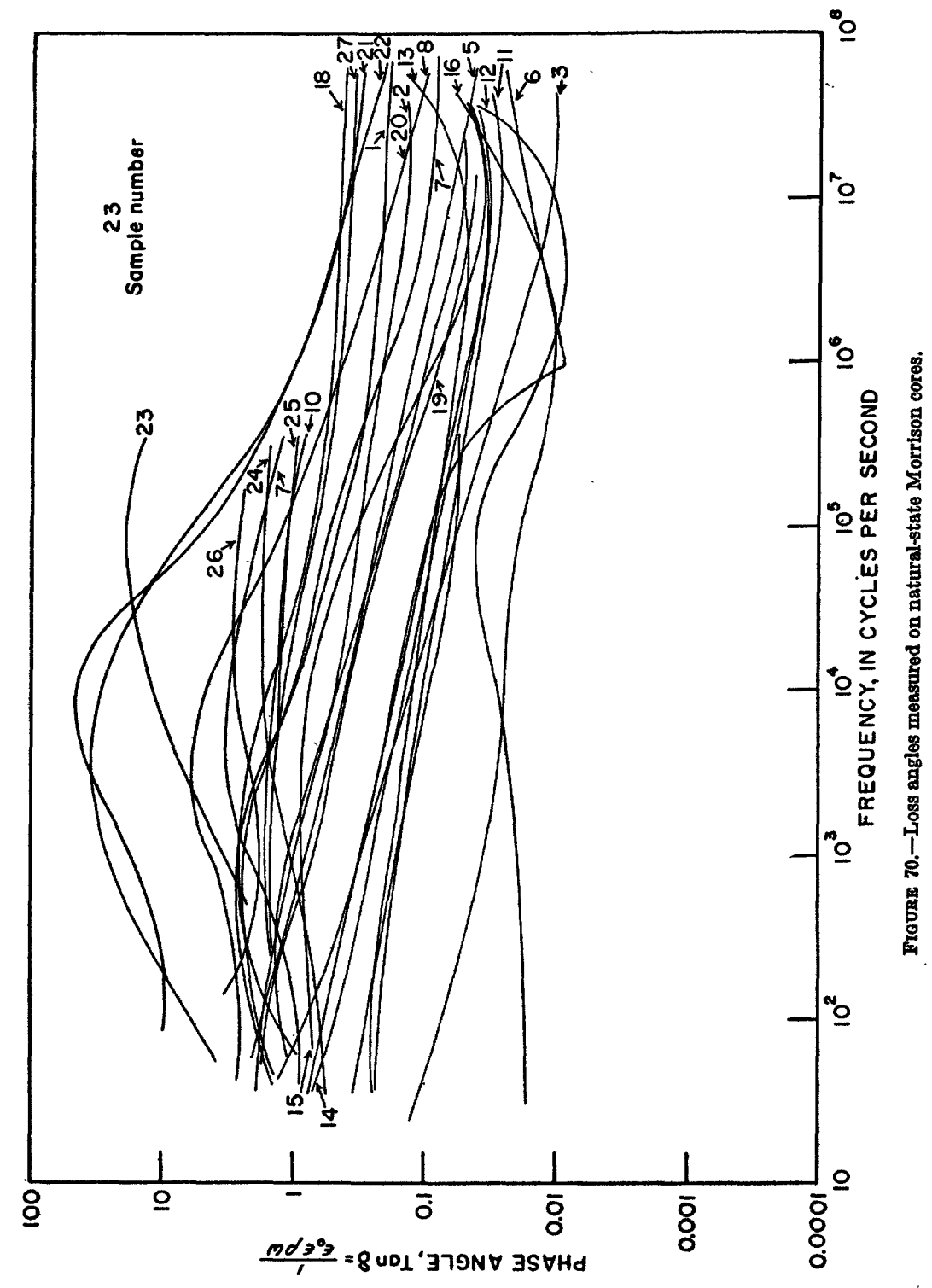




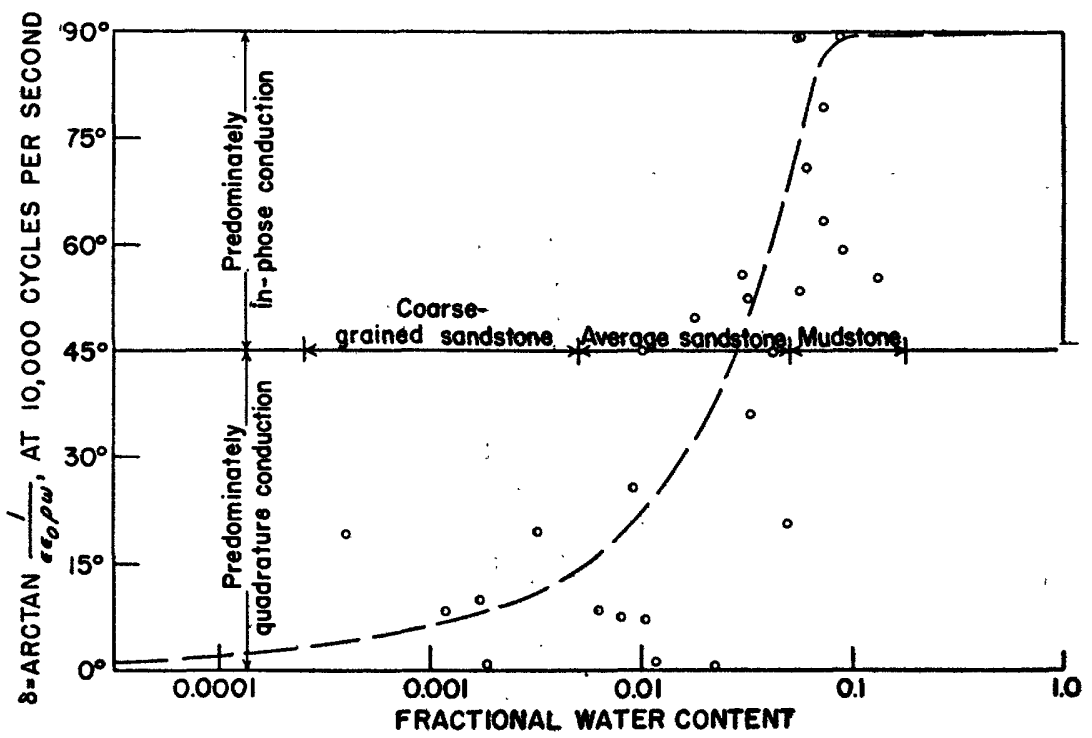

Figune 71.-Phase angle versus water content at a frequeney of $10 \mathrm{kc}$.

\section{MEASUREMENTS ON DRY CORES}

The behavior of resistivity and dielectric constant described in the preceding sections seems to be intimately associated with the presence of water in the cores. As a further check on this, measurements of dielectric constant and resistivity were made on the same group of cores after their fluid contents had been removed. The oil in the cores taken with oil-base mud was removed in a Soxhlet extractor with pentane. Most of the water was removed by baking the cores at $160^{\circ} \mathrm{C}$ for 72 hours. The cores were evacuated while still hot, resaturated with dry nitrogen, and then stored in a dry nitrogen atmosphere. Measurements were later made with the cores in a vacuum. This procedure ensured the removal of all water except that closely associated with minerals in the cores, and it prevented re-entrance of moisture from the atmosphere while measurements were being made.

Some dispersion is evident in the dielectric constants of the dry cores (fig. 72). The dielectric constants at low frequencies are as much as 20 percent more than those at the high frequencies, but the tremendous variations observed in the cores in their original state are absent. It is apparent that the observed dispersion was entirely dependent on the presence of water.

Determinations of resistivity and phase angle were made on these dried cores; but as might be expected, the ohmic conductivity is practically negligible. Because the in-phase current is such a small 


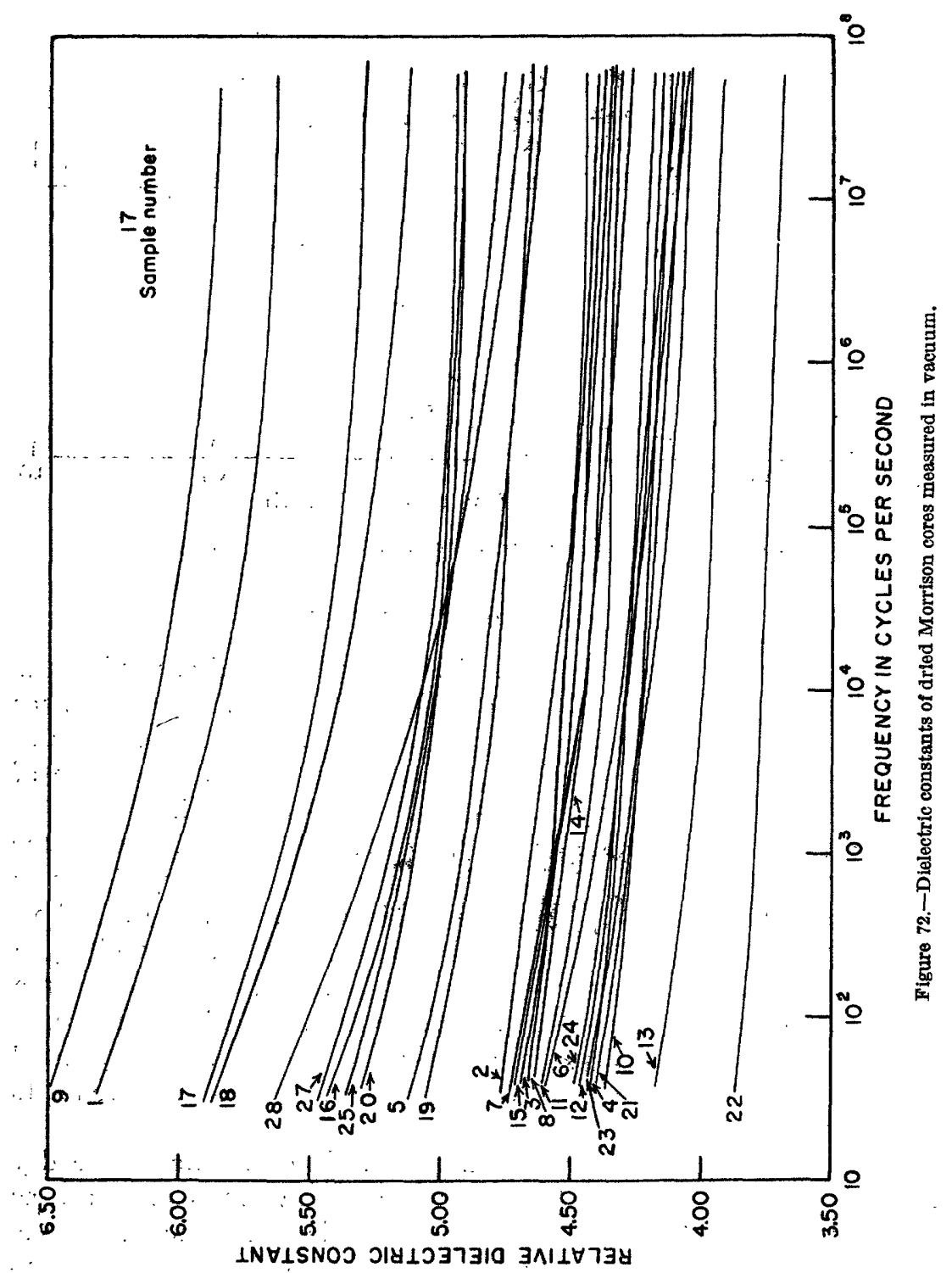


part of the total current in the dried cores, only a very low accuracy was achieved in these determinations. It was found, however, that all dried cores behaved as did cores number 10 and 12 in their natural state; that is, the resistivity of the dried cores was inversely proportional to the frequency.

\section{EVALUATION OF THE DATA IN TERMS OF DISPERSION THEORIES}

Examples of dispersion of electrical resistivity and dielectric constant are abundant in the literature; though few measurements of dielectric constants as high as those observed on some of the cores in the present work have been reported. The most pertinent of these earlier measurements are those of H. M. Evjen (1948), who estimated. a dielectric constant of $10^{8}$ to explain transients observed in a sedimentary section. Dielectric constants as large as $10^{3}$ or $10^{5}$ have been reported by Fricke $(1925,1926)$ for certain biological-cell suspensions, by Fricke and Curtis (1935) for kaolin-gelatin suspensions, by Koops (1951) for artifically prepared ferrites, by Von Hippel (1954) for bottom-round steak, by Dunlap and Makower (1945) for dehydrated carrots, and by Smith-Rose (1934) and Tarkhov (1948) for rocks and other earth materials.

Several explanations for such anomalous behavior of dielectric materials have been advanced by the various authors. Smith-Rose (1934) and Tarkhov (1948) have suggested that the high dielectric constants observed on moist rocks are caused by electrode polarization. It is a well-known fact that a pair of metallic electrodes immersed in an ionic solution will act as a condenser of large capacity. if current is caused to flow between the electrodes. This capacity is confined to a narrow zone near the electrode surface and is caused in part by a change in concentration near the electrodes during current flow, resulting in a change of the normal electrode potential in opposition to the direction of current flow. In addition, the inincreased density of cations near the cathode and of anions near the anode results in a concentration potential, also opposed to the current. flow. For steady-state direct currents, the capacity of a smooth electrode surface is of the order of 6 to 12 microfarads per square. centimeter. The capacity of metal electrodes with a large specific surface area may be much larger, and polarization capacities as large as 0.02 farads per square centimeter have been reported (Jaffe and Rider, 1952). With an alternating current, these reactions are effective only if the ions have time to concentrate at the electrodes during each half cycle. It can be shown theoretically that if the diffusion potential is the only factor contributing to the polarization capacity, then that capacity should decrease as the square root of 


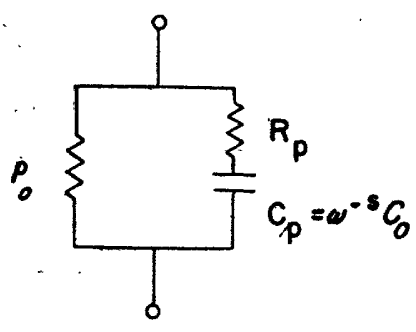

$$
\begin{aligned}
& \rho=\left(\frac{1}{\rho_{0}}+\frac{1}{R_{\rho}} \frac{C_{\rho^{2}} R_{\rho^{2} \omega^{2(1-s) ~}}}{1+C_{\rho^{2}} R_{\rho^{2}} \omega^{2(1-3)}}\right)^{-1} \\
& \epsilon=\frac{C_{p}}{1+C_{\rho^{2}} R_{\rho^{2}} \omega^{2(1-s)}}
\end{aligned}
$$

C.B. Jolliffe, 1923

Frgure 73.-Equivalon't eircuit for electrode polarization.

frequency. In a study of polarization capacity at intermediate and high frequencies, Jolliffe (1923) found that the inverse square-root relation held good for platinum electrodes. Other investigators have found that the capacity dies off less rapidly than the square root of frequency, and still others have found an inverse proportionality. Jolliffe (1923) has suggested that electrode polarization can be represented by the equivalent circuit shown in figure 73 . The effects of electrode polarization are represented by a resistor and by a variable capacitor in series and shunted with a second resistor. The capacitance is assumed to decrease exponentially as frequency increases, the factor $s$ representing the rate of decrease. Such an equivalent circuit would result in an apparent dispersion of the dielectric constants as shown by the curves presented in figure 74 . For comparison, one of the observed dielectric-dispersion curves is shown. It is apparent that the observed dielectric constant decreases less rapidly with frequency than any of the cases where the dispersion might be the result of electrode polarization.

Further evidence that the high dielectric constants are not caused by electrode polarization is given by extrapolating dielectric constants to zero frequency. The apparent dielectric constant that would result from an electrode polarization capacity of 10 microfarads per square centimeter is shown as a function of water content by the solid curve in figure 75 . The observed values of static dielectric constant are 2 to 5 orders of magnitude smaller than would be expected if the dispersion were caused by electrode polarization. This indicates that in the frequency range used in the present work, the effects of electrode polarization are relatively unimportant.

Actually, all solid and liquid dielectrics possess abnormal or anomalous properties at low frequencies beyond those that can be explained by electrode polarization (Hartshorn, 1926). In addition to the normal charging current and conduction current, such dielectrics exhibit an anomalous charging current when subjected to a steady-state 


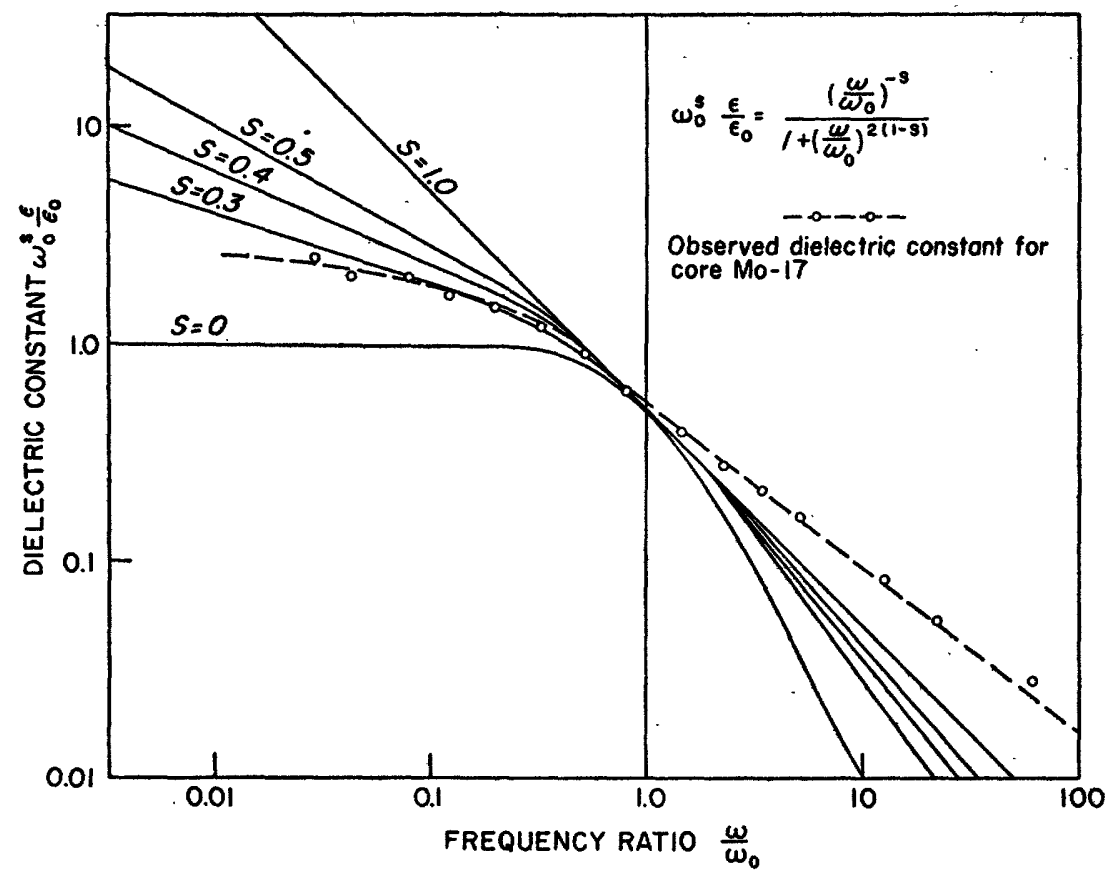

FrgURE 74.-Dispersion curves showing effect of electrode-polarization capacity decaying as an inverse power of the frequency.

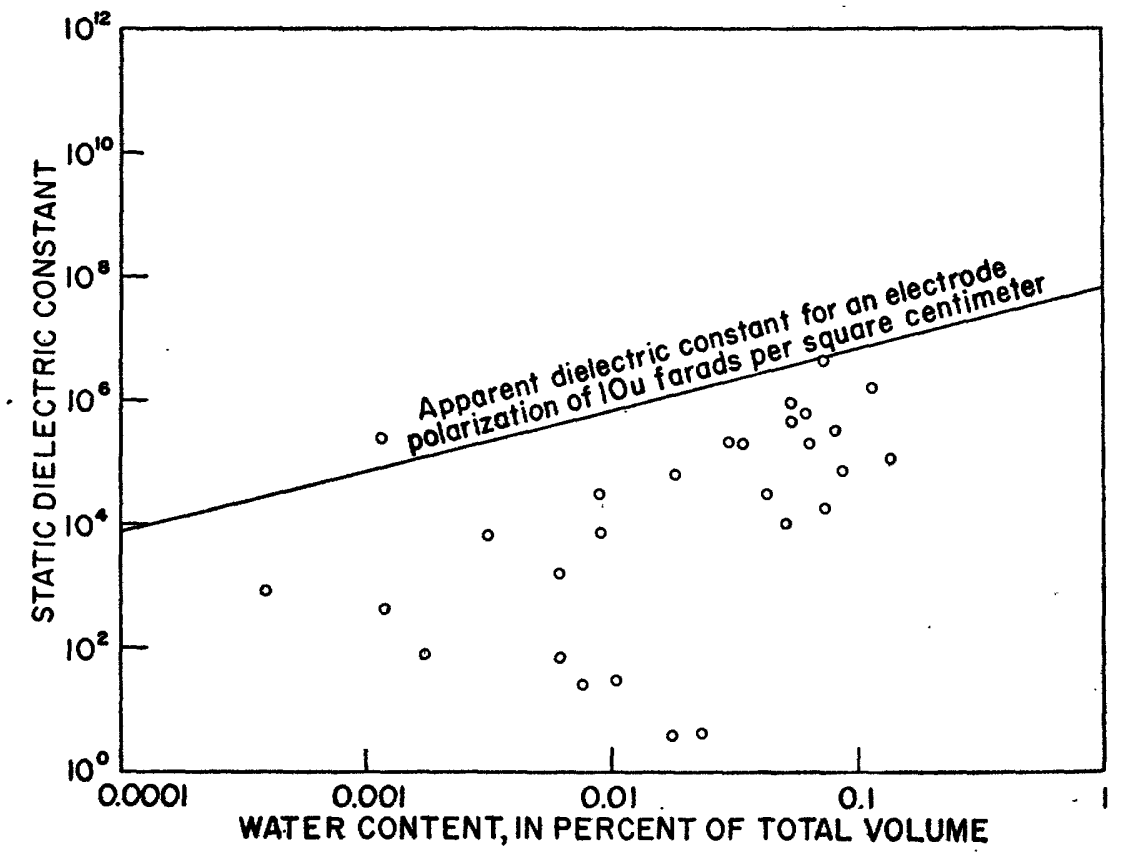

FrguRe 75.-Static dielectric constant versus water content. 
voltage. When a static voltage is applied to a material with anomalous behavior, the current flowing may be expressed as

where

$$
I_{t}=i_{0}+i_{1}(t)+i_{2}(t)
$$

$$
\begin{aligned}
i_{0} & =\text { the ohmic current flowing in the medium } \\
i_{1}(t) & =\text { the normal capacitative charging current } \\
i_{2}(t) & =\text { the anomalous charging current }
\end{aligned}
$$

This anomalous charging current is comparatively large at the instant the voltage is applied but decreases rapidly with time. However in some materials an appreciable part of this current may persist for months. As the anomalous current is proportional to the applied voltage, its characteristics may be expressed by the equation

where

$$
i_{2}(t)=C_{0} E \beta F(t)
$$

$C_{0}=$ the geometric capacity of a system

$E=$ the applied voltage

$\beta=a$ proportionality parameter specifying the magnitude of the anomalous current in a particular material

$F(t)=$ the characteristic time function, describing the manner in which the anomalous current decreases with time

The form of $F(t)$ is a matter of some dispute. Many early investigators found that within the limits of their measurements the characteristic time function was a simple power function

$$
F(t)=\beta t^{-m}, m<1
$$

It is obvious, though, that this expression cannot be physically correct, because if one considers zero time, the current is infinite, and if one considers the accumulated charge during an infinite charging period, this is also infinite.

In an alternating field the anomalous current will increase both the apparent capacity and the loss; the amount of this increase depends on the length of a cycle. According to Hartshorn (1926) Schweidler has shown that the fractional increase in capacity caused by the anomalous current is

where

$$
\Delta C / C=\beta \int_{0}^{\infty} F(t) \cos \omega t d t
$$

$\omega=$ the angular frequency of the alternating field

An exponential form for $F^{\prime}(t)$

$$
F(t)=\beta e^{-\alpha t}
$$

has been suggested on theoretical grounds by Maxwell and by Pellat (Hartshorn, 1926). Maxwell arrived at this expression by considering 
the discharge current from a material composed of alternating layers of different electrical properties, and Pellat arrived at the same result by considering a viscous displacement of charge following the initial elastic deformation in an applied field.

Unfortunately, observed data do not ordinarily follow simple exponential time functions. Schweidler has extended Pellat's analysis by considering a characteristic time function consisting of a sum of exponential terms

$$
F(t)=\Sigma \beta_{n} e^{-\alpha_{n} t}
$$

where there is an infinite variety of arbitrary coefficients $\beta_{n}, \alpha_{n}$.

This equation may be applied in a practical case by specifying a law of distribution for these arbitrary factors. Several such laws have been suggested (Fuoss and Kirkwood, 1941; Macdonald, 1952; and Davidson and Cole, 1950), but the law which is most applicable to our data is a log-normal distribution law suggested by Wagner (1913). Wagner's equation for the characteristic time function is

$$
F(t)=\int_{0}^{\infty} \gamma(T) e^{-\frac{t}{T}} d T
$$

where

$\gamma(T) d T=$ the sum of the amplitudes of all terms in the range from $T$ to $d T$, with the distribution function replacing the individual amplitude parameters $\boldsymbol{\beta}_{\boldsymbol{n}}$ of equation 7

$T=$ relaxation time, replacing the reciprocals of the parameters $\alpha_{n}$ in equation 7

For a log-normal distribution, the distribution function is

$$
\gamma(T) d T=\frac{\gamma b e^{-b^{2} z^{2}}}{\sqrt{\pi}} d z ; z=e n \frac{T}{T_{0}}
$$

where

$T_{0}=$ the principle value of $T$, the time constants

$b=a$ parameter expressing how closely the values of the time constants are grouped about their principle value

Curves showing the distribution of time constants for various values of $b$ are presented in figure 76 .

Wagner has shown that with such a distribution of time constants, the capacity and phase angle of a material are given by the following equations:

$$
\begin{gathered}
\frac{\Delta C}{C}=\frac{\gamma b e^{-b^{2} z_{0}^{2}}}{\sqrt{\pi}} \int_{0}^{\infty} e^{-b^{2} u^{2}} \frac{\cosh \left(2 b^{2} z_{0}-1\right) u}{\cosh u} d u \\
\left(1+\frac{\Delta C}{C}\right) \tan \delta=\frac{\gamma b e^{-b^{2} z_{0}^{2}}}{\sqrt{\pi}} \int_{0}^{\infty} e^{-b^{2} t^{2}} \frac{\cosh b^{2} z_{0} u}{\cosh u} d u
\end{gathered}
$$

$493911-59-3$ 
where

$$
\begin{aligned}
z & =\ln T / T_{0} \\
z_{0} & =\ln \omega T_{0} \\
z+z_{0} & =u
\end{aligned}
$$

Curves for this form of dielectric dispersion are presented in figure 77, and curves for the variation of phase angle with frequency are presented in figure 78.

An adaptation of a graphical method described by Yager (1936) was used to determine the parameters $b, \gamma$, and $T_{0}$ for the dispersion data obtained with the natural-state Morrison cores. In this technique a set of curves relating a factor $\delta$ (defined as the ratio of two values of dielectric constant, one at 10 times the frequency of the other) a factor $z=\ln T_{0} / T_{r}$ ( $T_{0}$ is the principal relaxation time, and $T_{r}$ is the relaxation time for the frequency at which $\delta$ is defined) and the distribution parameter $b$ are used. These curves are presented in figure 79 .

In analyzing a particular group of data, the ratio of two dielectric constants a decade apart in frequency is formed; and by using the curves in figure 79, a curve relating $z$ and $b$ is constructed. This process is repeated with other pairs of dielectric constants taken at other frequencies. The final result is a series of curves of $z$ versus $b$ for a particular set of dielectric constants, as shown by the example

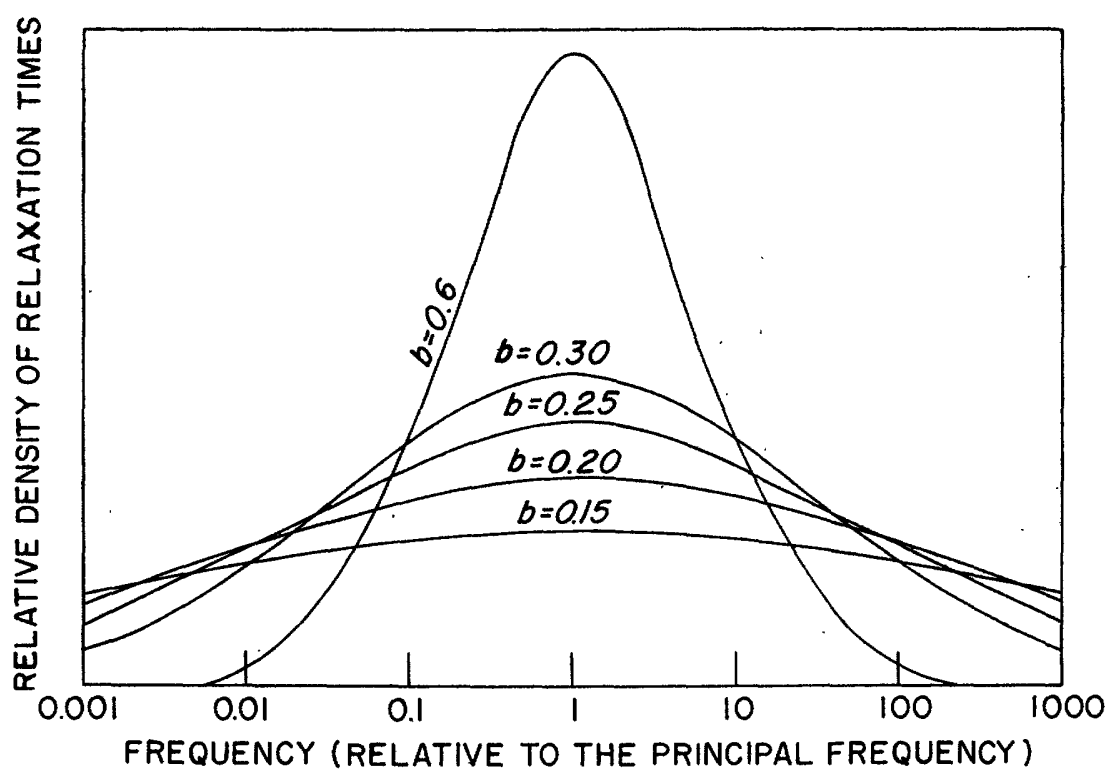

FIGURE 76.-Distribution of relaxation times as a function of the distribution parameter $b$. 


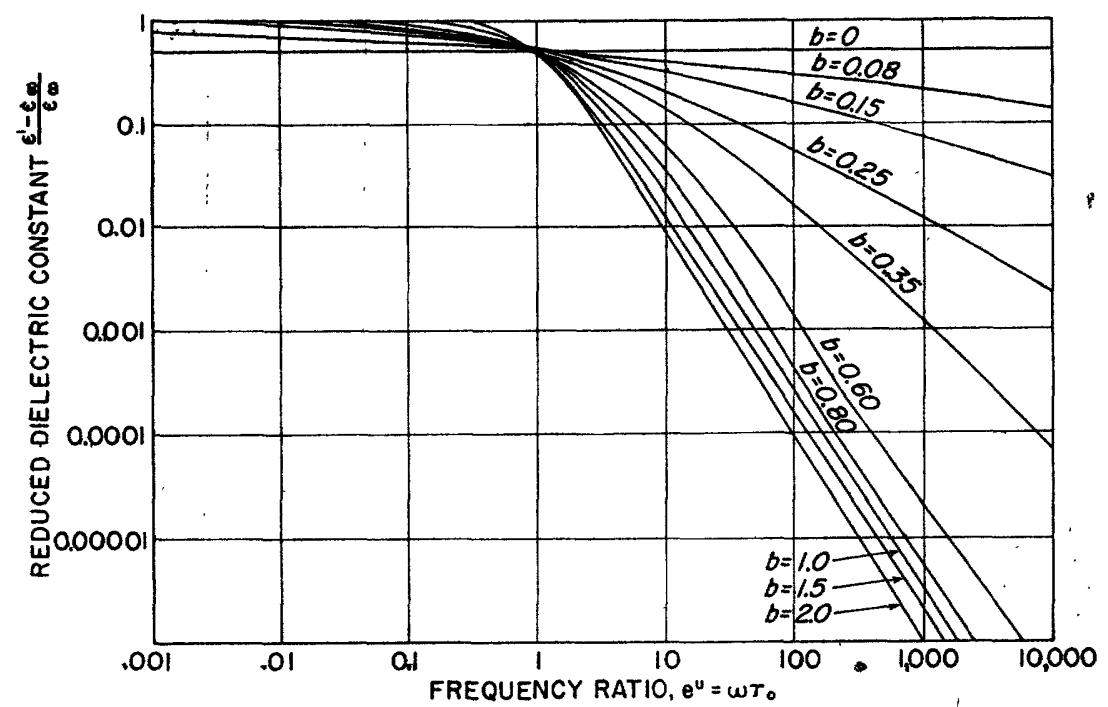

Fiqure 77.-Wagner's equation for dispersion of the real dielect:ic constant in a substance with a normal distribution of relaxation times.

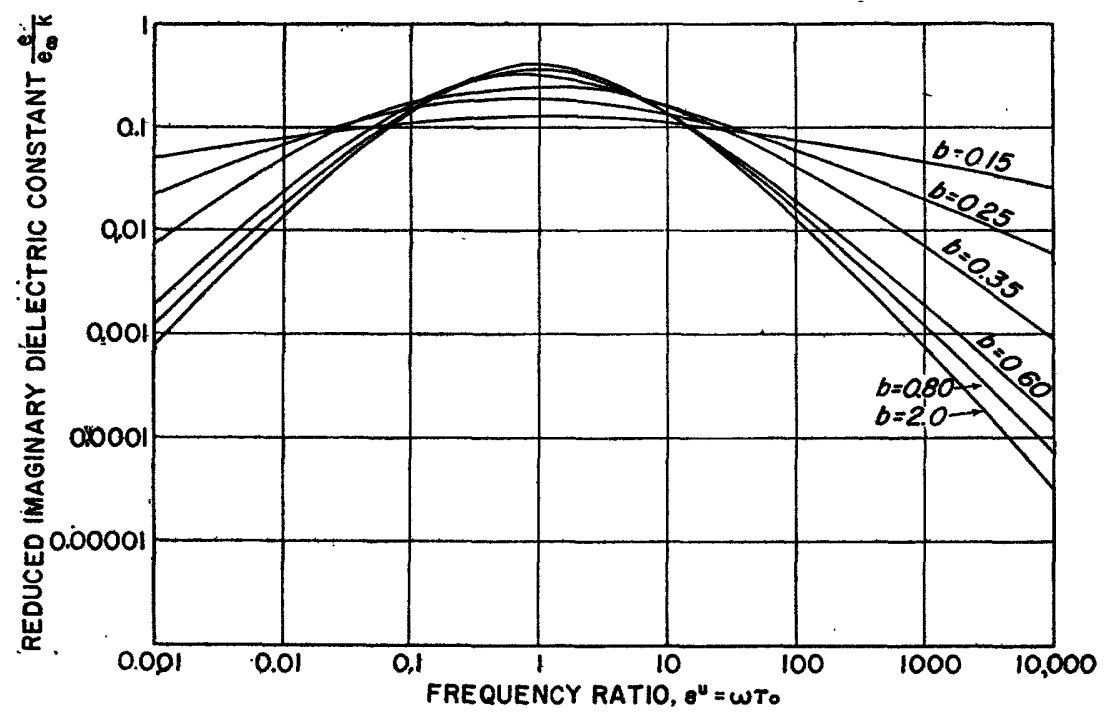

Figure 78.-Equation for dispersion of the imaginary dielectric constant in a substance with a normal distribution of relaxation times. 


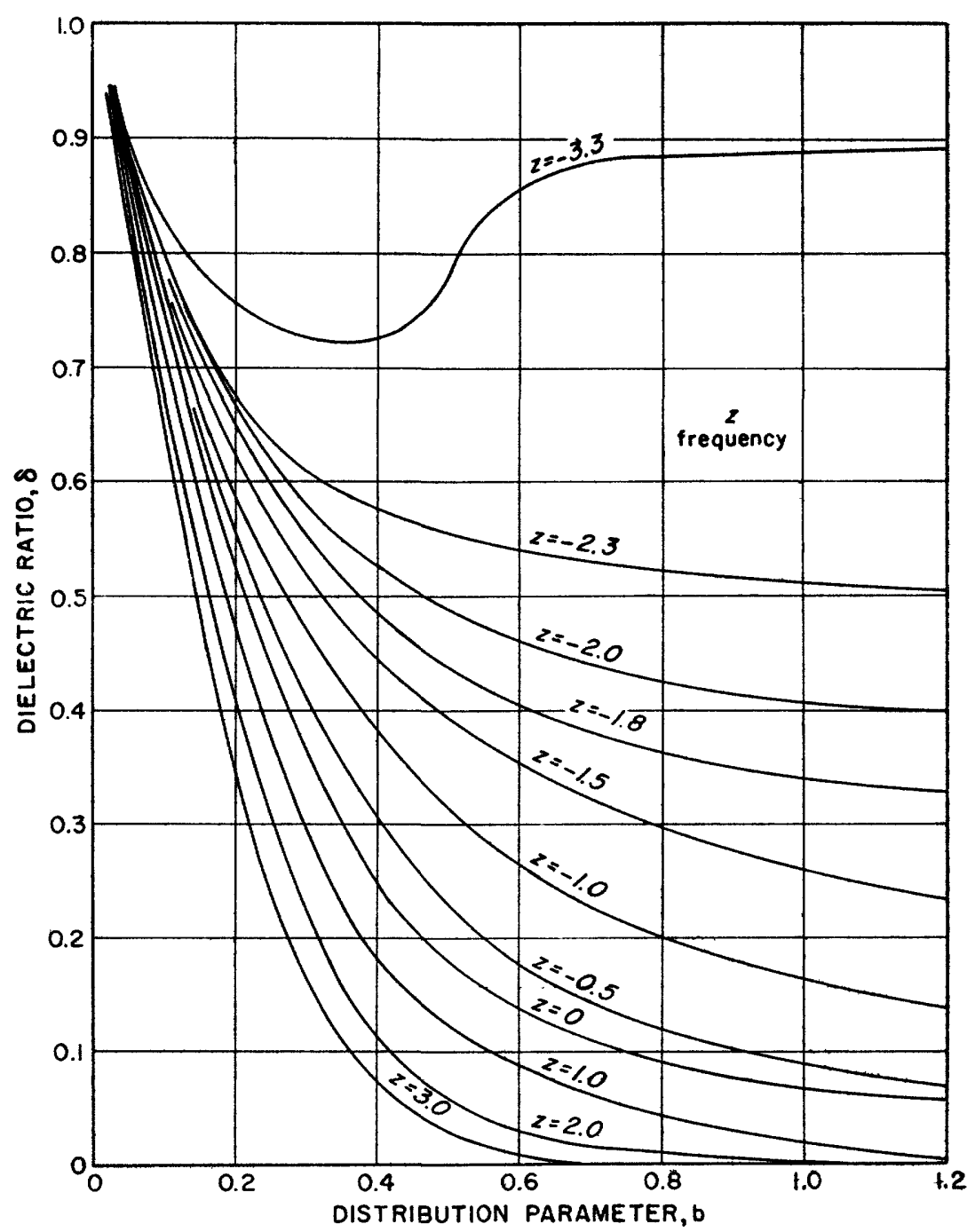

Figune 79.-Dielectric ratio as a function of distribution parameter and frequency.

in figure 80. The intersection of these tria] curves determines the correct values of $b$ and $z_{0}$ for the data. The principal relaxation frequency may be obtained from the definition

where

$$
z_{0}=\ln \omega_{r} / \omega_{0}
$$

$\omega_{r}=$ the frequency at which the first dielectric ratio was formed $\omega_{0}=$ the principal relaxation frequency 


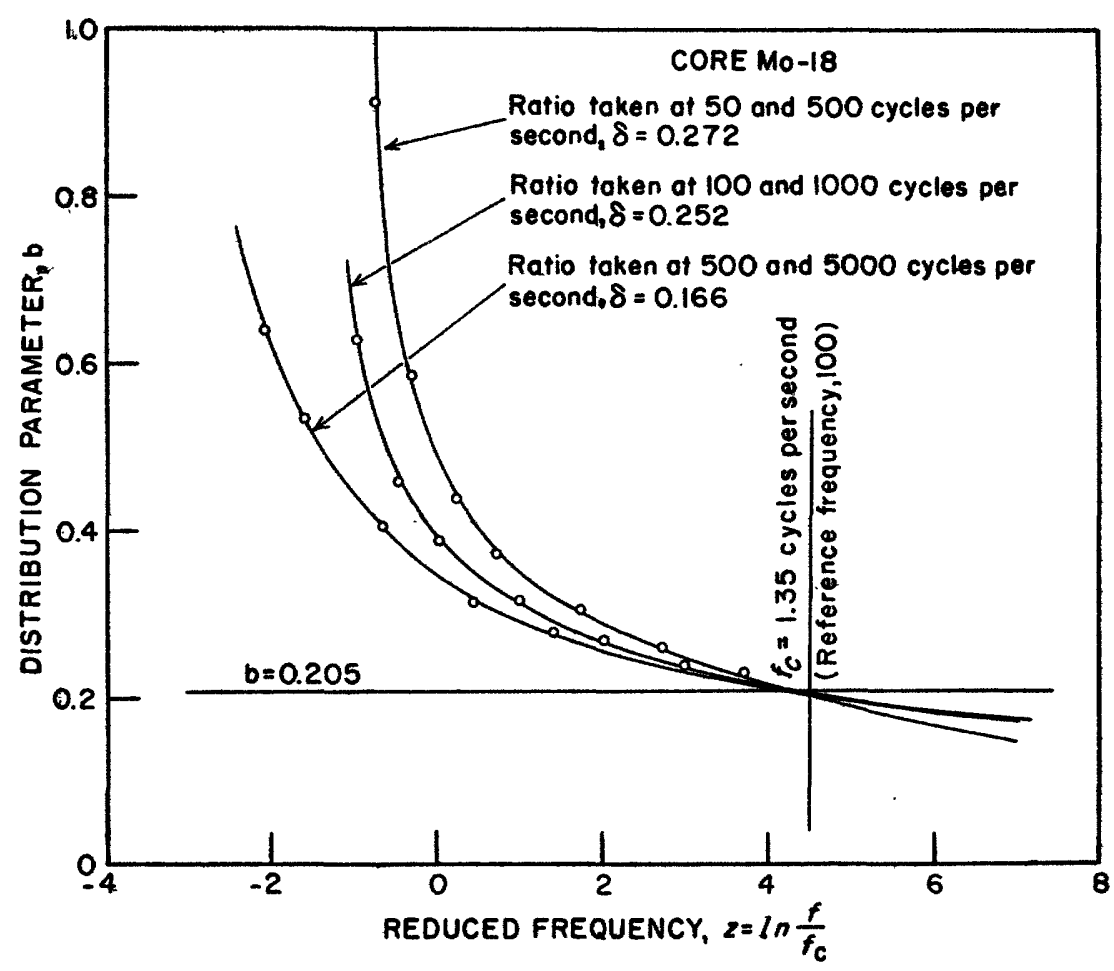

Freore 80.- Fxample of the graphical determination of Wagner's parameters $b$ and $z_{0}$.

The second stage in the graphical evaluation of the data is the determination of the low-frequency or static value of dielectric constant. For this purpose a group of curves showing the relation between $b$ (the distribution parameter), $z=\ln \omega / \omega_{0}$ (the frequency), and the dielectric constant (expressed as a fraction of the maximum value attained at low frequencies) is used. These curves are presented in figure 81 .

As $b$ and $z$ are already known from the analysis described in the preceding paragraph, it is a simple matter to determine a theoretical value for the dielectric constant from figure 81 for each of the frequencies at which an experimental value was determined. When the experimental values are plotted as a function of these theoretical values, a straight line relation with a slope equal to the low-frequency dielectric constant is obtained provided the data satisfy Wagner's equations. An example is shown in figure 82 .

This procedure was used to determine the dispersion parameters for each of the cores studied. The results are given in table 1 . 


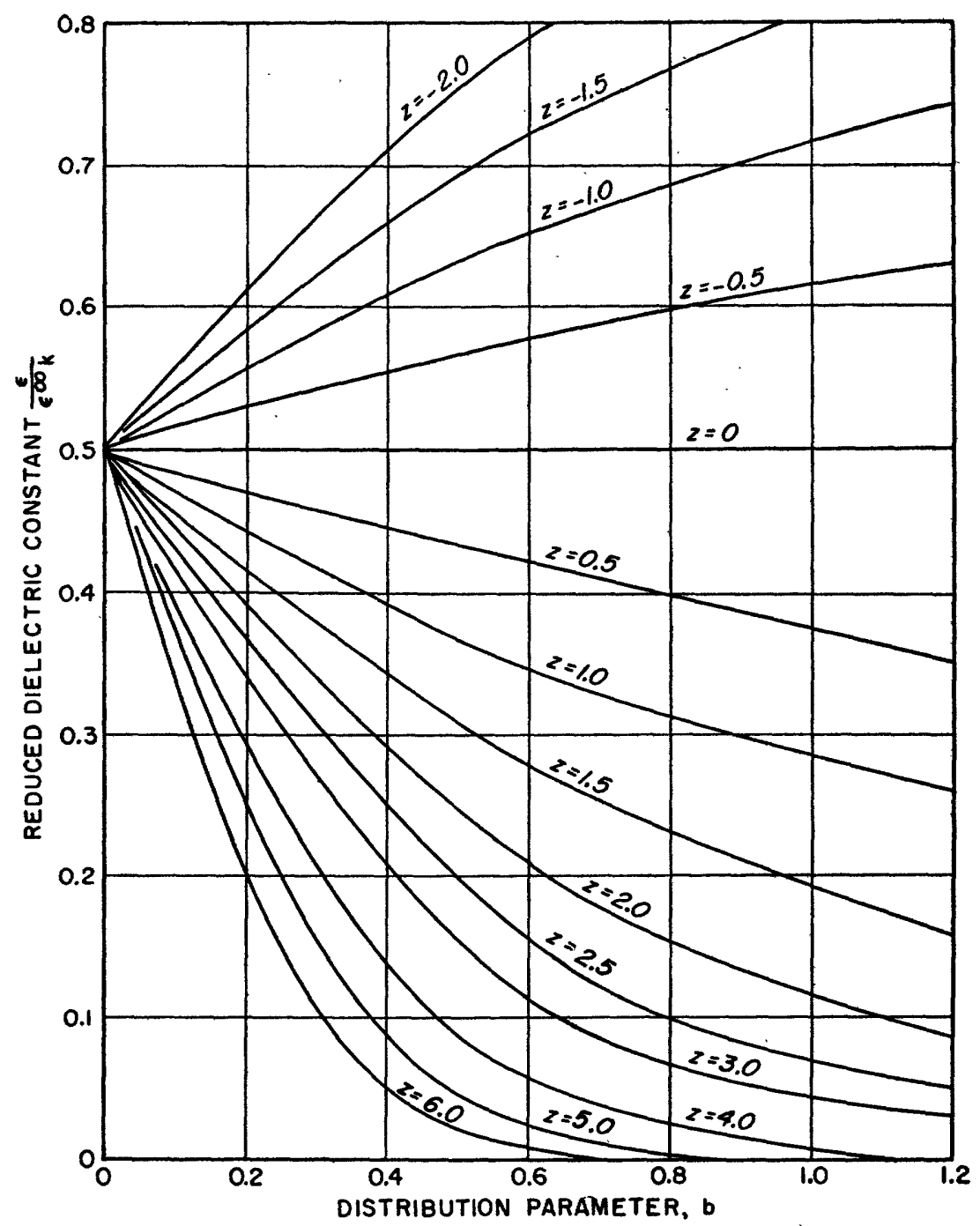

FIGURE 81.-Theoretical dielectric constant as a function of distribution parameter and frequency.

An example of the agreement between the experimental data and Wagner's equations is given in figure 83 . The observed values of resistivity are plotted in terms of an imaginary dielectric constant, defined as

where

$$
\epsilon^{\prime \prime}=\frac{i \omega \rho}{\epsilon_{0}}
$$

$\omega=$ the frequency in radians per second

$\rho=$ the electrical resistivity in ohm-centimeters

$\epsilon_{0}=$ the dielectric constant of a vacuum in farads per centimeter 


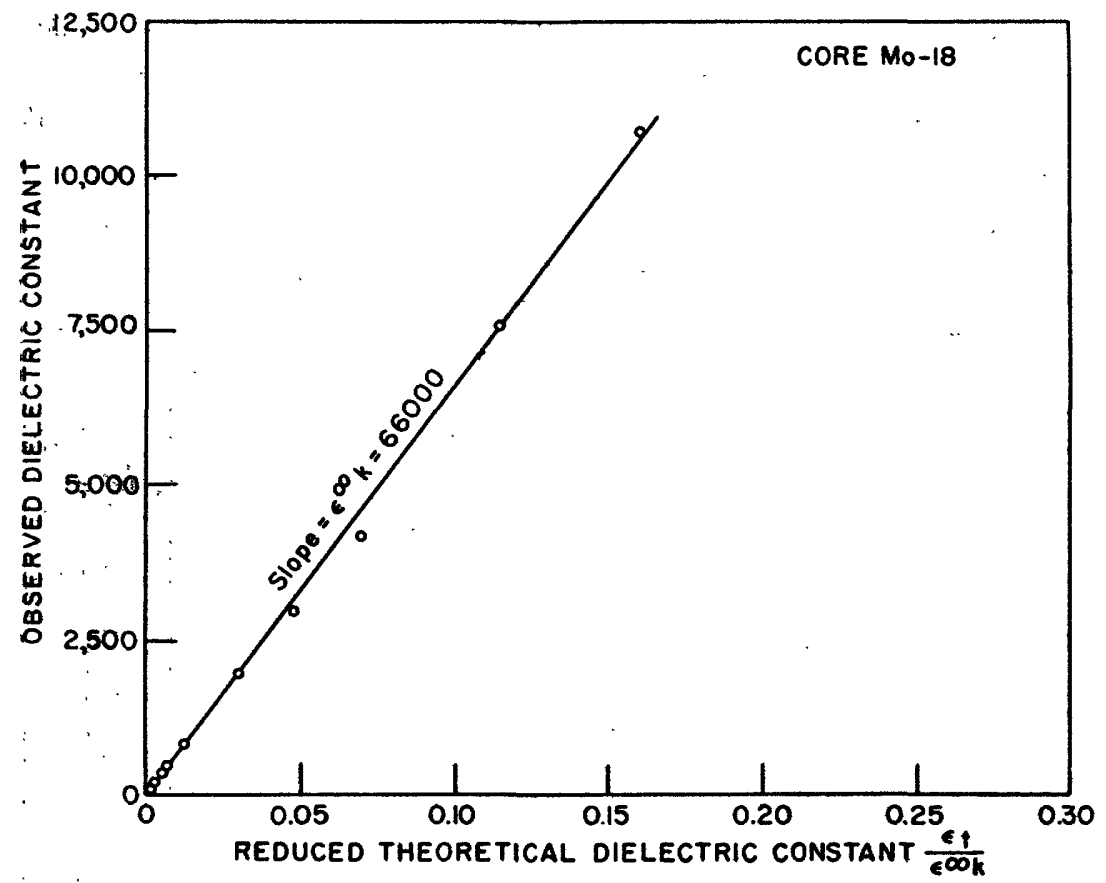

FJGURe 22,-Graphical determination of the maximum dielectric constant and the parameter $k$ of Wagner's equation.

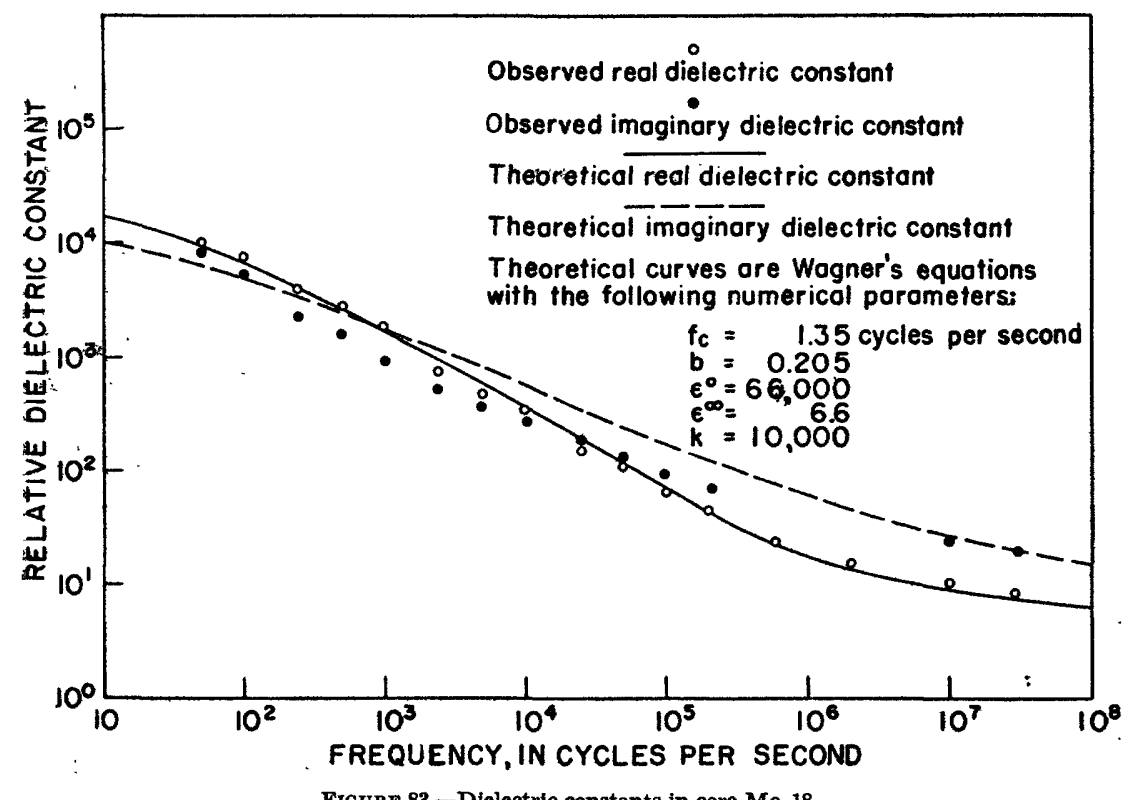

FIGURE 83.-Dielectric constants in core Mo-18. 
The agreement between the real dielectric constant and Wagner's equation was excellent in all cases, but the agreement between imaginary dielectric constant (resistivity) and theoretical curves is not good.

TABLE 1.-Characteristics of the dielectric dispersion of natural-state cores

\begin{tabular}{|c|c|c|c|c|c|c|}
\hline Core No. & $\begin{array}{l}\text { Water } 1 \\
\text { content }\end{array}$ & $\begin{array}{l}\text { Relaxation } \\
\text { frequency }\end{array}$ & $\begin{array}{l}\text { Distribu- } \\
\text { tlon par- } \\
\text { ameter } b\end{array}$ & $\begin{array}{c}\text { Low- } \\
\text { frequency } \\
\text { dielectric } \\
\text { constant }\end{array}$ & $\begin{array}{l}\text { High- } \\
\text { frequency } \\
\text { dielectric } \\
\text { constant }\end{array}$ & $\begin{array}{c}\text { Dielectric } \\
\text { increaso } \\
\text { ratio }\end{array}$ \\
\hline $\begin{array}{l}1 \mathrm{~A} \\
\mathrm{3} \\
\mathrm{4}\end{array}$ & $\begin{array}{l}0.088 \\
.0089 \\
.0012 \\
.0114 \\
.0031 \\
.00179 \\
.0004 \\
.00103 \\
.0609 \\
.0189 \\
.0106 \\
.0240 \\
.00796 \\
.00618 \\
.0340 \\
.0316 \\
.0613 \\
.0180 \\
.0519 \\
.0730 \\
.0545 \\
.0556 \\
.0833 \\
.0415 \\
.135 \\
.0728 \\
.0306\end{array}$ & $\begin{array}{c}61.3 \\
1.75 \\
18.254 \\
18.1 \\
1.37 \\
2.60 \\
.87 \\
.68 \\
.338 \\
1.29 \\
1.01 \\
.742 \\
11.2 \\
55.3 \\
1.35 \\
1.331 \\
1,510 . \\
670 . \\
2,470 . \\
4,589 . \\
2.27 \\
12.2 \\
.308 \\
2.23\end{array}$ & $\begin{array}{l}0.24 \\
.26 \\
.16 \\
.24 \\
.295 \\
.20 \\
.20 \\
.275 \\
.20 \\
0 \\
.135 \\
0 \\
.125 \\
.175 \\
.145 \\
.35 \\
.25 \\
.205 \\
.24 \\
.60 \\
.50 \\
.76 \\
.72 \\
.25 \\
.29 \\
.16 \\
.27\end{array}$ & \begin{tabular}{l}
$7.0 \times 10^{4}$ \\
$8.0 \times 10^{3}$ \\
$3.95 \times 10^{2}$ \\
$1.58 \times 10^{6}$ \\
$7.1 \times 10^{3}$ \\
76 \\
$8.65 \times 10^{3}$ \\
$2.45 \times 10^{4}$ \\
$6.0 \times 10^{5}$ \\
\hdashline 32 \\
\hdashline 28 \\
72 \\
$1.41 \times 10^{3}$ \\
$2.10 \times 10^{5}$ \\
$1.94 \times 10^{5}$ \\
$6.3 \times 10^{4}$ \\
$1.10 \times 10^{4}$ \\
$1.63 \times 10^{4}$ \\
$8.4 \times 10^{5}$ \\
$4.3 \times 10^{3}$ \\
$2.88 \times 10^{5}$ \\
$2.90 \times 10^{4}$ \\
$1.04 \times 10^{5}$ \\
$3.75 \times 10^{6}$ \\
$1.96 \times 10^{5}$
\end{tabular} & $\begin{array}{l}10.0 \\
6.9 \\
3.8 \\
3.56 \\
9.0 \\
3.67 \\
4.65 \\
6.1 \\
7.9 \\
4.2 \\
3.6 \\
4.3 \\
3.75 \\
4.0 \\
4.6 \\
9.0 \\
9.0 \\
7.1 \\
5.6 \\
5.7 \\
7.5 \\
5.3 \\
6.9 \\
6.6 \\
8.3 \\
6.1 \\
6.7\end{array}$ & $\begin{array}{c}7.0 \times 10^{2} \\
1.16 \times 10^{3} \\
104 \\
4.43 \times 10^{5} \\
7.9 \times 10^{4} \\
21 \\
188 \\
4.0 \times 10^{4} \\
7.6 \times 10^{4} \\
1.0 \\
8.9 \\
1.0 \\
7.5 \\
18.1 \\
309 \\
2.33 \times 10^{4} \\
2.15 \times 10^{4} \\
8.9 \times 10^{3} \\
1.97 \times 10^{3} \\
2.86 \times 10^{2} \\
1.12 \times 10^{5} \\
8.1 \times 10^{4} \\
4.17 \times 10^{4} \\
4.39 \times 10^{3} \\
1.25 \times 10^{4} \\
6.13 \times 10^{5} \\
2.88 \times 10^{4}\end{array}$ \\
\hline
\end{tabular}

1 Determined by U.S. Bureau of Mines, San Francisco, Calif.

Wagner's equations have provided a satisfactory description of dispersion of the dielectric constant and resistivity, but the problem of constructing a pragmatic model that can explain the physical processes involved remains.

Maxwell (1892) has shown that anomalous phenomena can result from a particular megascopic structure of the dielectric material. If a material is composed of alternating layers of two materials with different electrical properties, these layers may be represented by the equivalent circuit shown in figure 84 . The increase in low frequency dielectric constant over the high frequency constant for such a system is given by

$$
K=\frac{\left(\tau_{1}+\tau_{2}-\tau\right) \tau-\tau_{1} \tau_{2}}{\tau_{1} \tau_{2}}
$$

where

$\tau_{1}=R_{1} C_{1}$

$\tau_{2}=R_{2} C_{2}$

$\tau=R_{1} R_{2}\left(C_{1}+C_{2}\right) /\left(R_{1}+R_{2}\right)$ and $R_{1}, R_{2}, C_{1}, C_{2}$ are the resistances and capacities of the alternate layers 


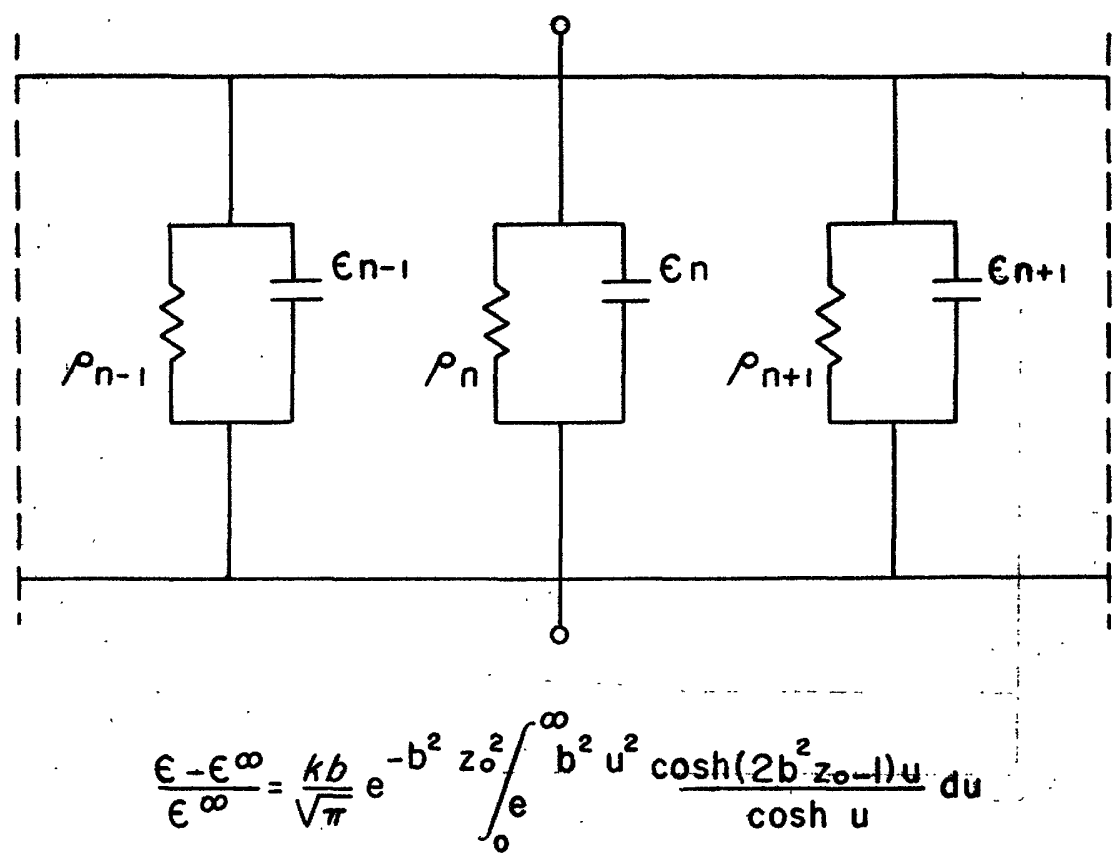

Wagner, 1913, Macdonald, 1952

FIGURE 84.-Equivalent circuit for a dielectric material having more than one relaxation time.

The ratio of the increase has been plotted as a function of water content for a hypothetical rock consisting of layers of quartz and water (fig. 85). Although Maxwell's interfacial polarization can account for large dielectric constants, these constants are found only at very large water contents.

Von Hippel (1954) points out that there is a tacit assumption in Maxwell's derivation that the migration of charge carriers (ions) through the layers is unimpeded. McCardell (McCardell and Winsauer, 1953) has suggested that mudstones or clay-bearing sandstones actually act as ion sieves. If a rock is considered to be a semipermeable membrane with fixed negative charges scattered through it, then it is expected that positive ions may flow through the rock more readily than negative ions. The characteristics of such selectively permeable membranes have been discussed by Sollner (1945).

A typical pore structure in a clastic detrital rock might be considered as a relatively.large, possibly equidimensional void, connected 


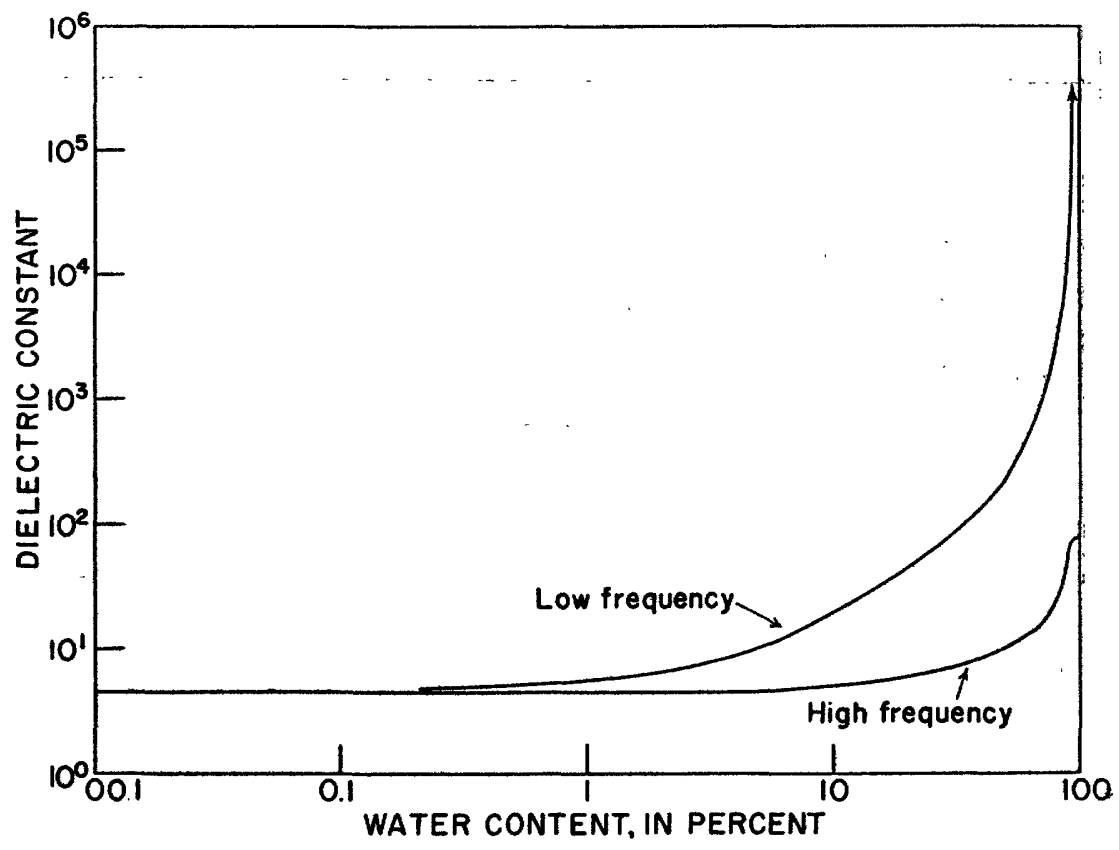

Fioukn 85.-Dielectric constant for a laminated mixture of quartz and water.

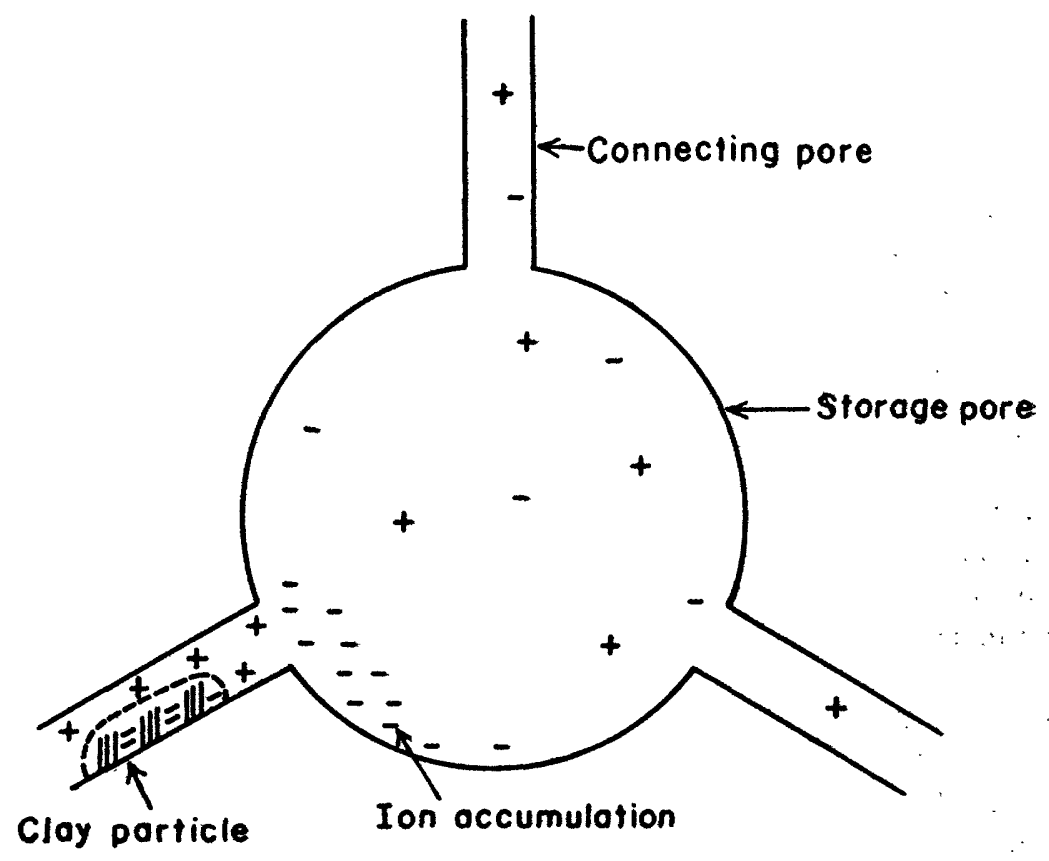

FToukr 86.-Idealized pore structure. 
to other such voids by thin fibrous channels. The essential features of such a pore structure are shown by the idealized "porelet" in figure 86. An example of the actual pore structure in a sandstone of the Morrison formation is shown in figure 87.

Clay particles positioned in constrictions in connecting channels will effectively block migration of negative ions because of the small size of the channels and the force of repulsions from the negative charges usually present on the clay particles. In such a situation the positive ions are free to move through the rock under the influence of an external field, but anions can migrate only until they come in contact with one of these selectively permeable pore constrictions. As a result there is an accumulation of negative charge as the anions

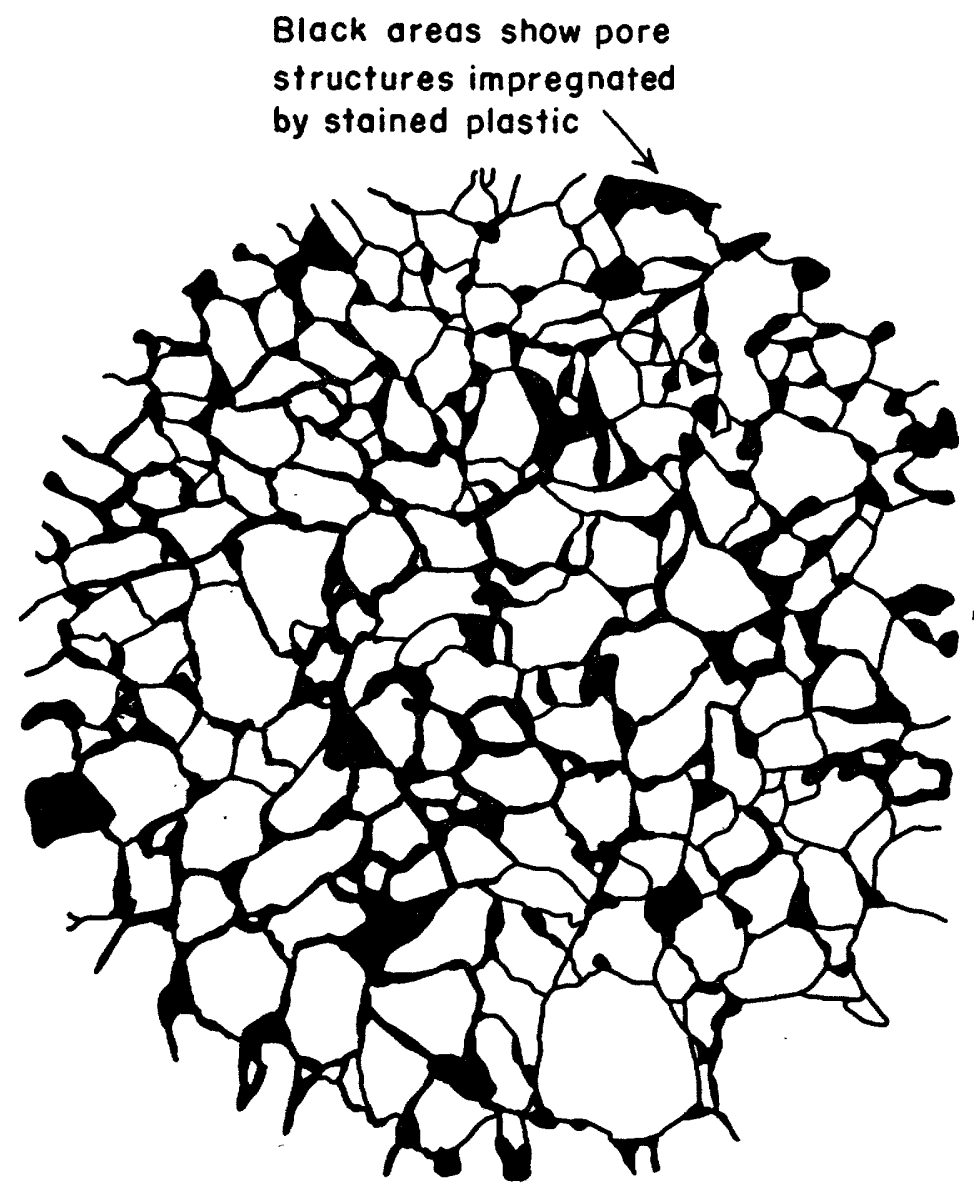

Frovar 87.-Tracing made from a photomicrograph of a stained thin section, showing the pore structure of a typleal sandstono in the Salt Wash member. 
migrate from one side to the other of the free area in a pore. The average anion would migrate a distance equal to

where

$$
d_{-}=\mu-\int_{t=0}^{t=t_{1}} \frac{V_{1}(t)}{D} d t
$$

$$
\begin{aligned}
\mu_{-} & =\text {the mobility of anions } \\
V_{1}(t) & =\text { the voltage drop across a given pore } \\
D & =\text { the diameter of that pore } \\
t & =\text { the length of time that voltage is applied }
\end{aligned}
$$

The voltage drop across a pore would be a function of time because of the counter electromotive force set up by the concentration differences as anions collect at the barriers. The diffusion potential set up by the anion concentrations will cause an accumulation of positive ions and an associated polarization; but as long as the diffusion potential is small compared to the applied voltage drop, the polarization due to cation concentration will be small compared to the polarization of the anions.

If it is assumed that the cations are relatively unaffected by the anion concentrations, the charge stored by the anions in one porelet will be

where

$$
q=\frac{d_{-}}{D} I_{-} t
$$

$I_{-} t=$ the total quantity of electricity passing through the pore by means of anion motion

$\frac{d-}{D}=$ the fraction of that motion which is not compensated by motion of cations in the opposite direction.

The fraction of the total current carried by the anions is a function of the ionic mobilities and the relative concentrations of cations and anions. The clay particles act as immobile anions, so the number of mobile anions will be less than the number of cations. The part of the total current carried by anions will be

where

$$
I_{-}=\frac{\mu_{-}}{\mu_{+}+\mu_{-}} \frac{\alpha-\alpha_{0}}{2 \alpha-\alpha_{0}} I
$$

$\mu_{+}$and $\mu_{-}=$the mobilities of the cations and anions, respectively

$\alpha=$ the concentration of cations

$\alpha_{0}=$ the equivalent concentration of the clay anions

Values of $a_{0}$ measured on a group of typical sandstones of the Morrison formation, fully water saturated, are presented in figure 88. 


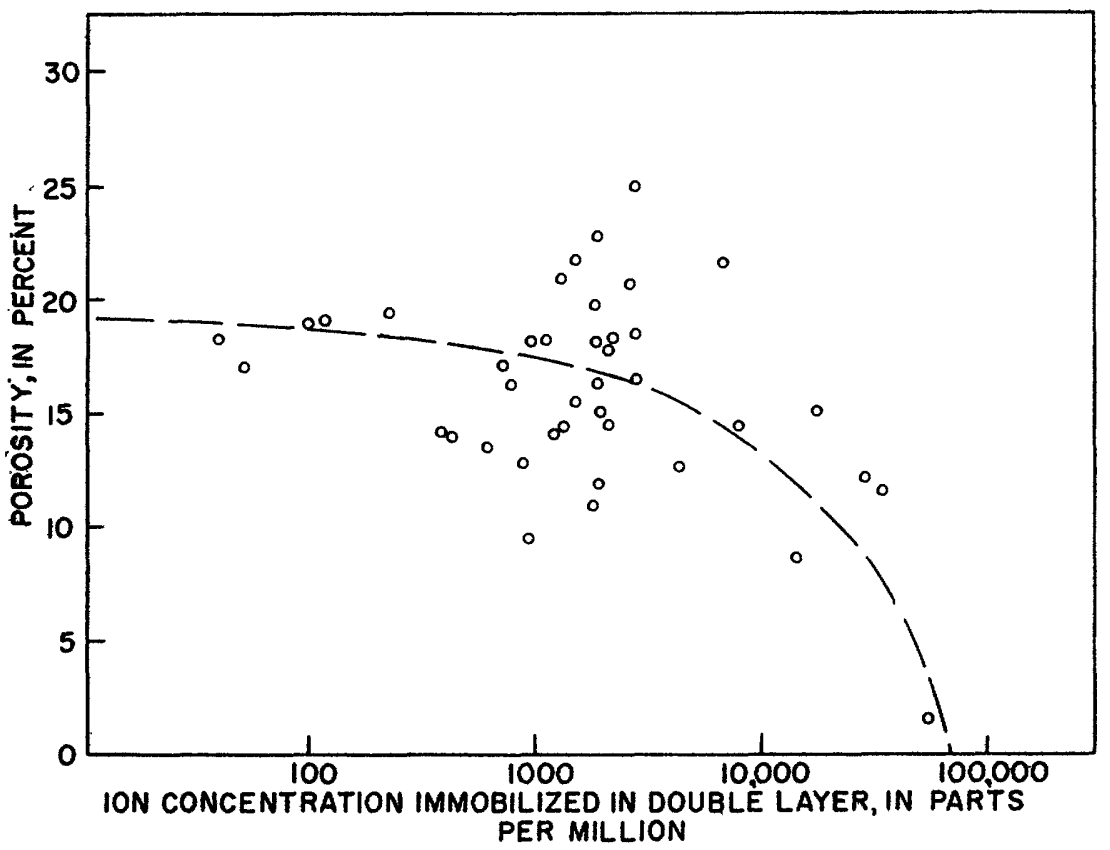

FraoRz 88.-Number of immobile cations in a typical sandstone of the Balt Wash member.

The volume of the central part of a pore, as presented in the idealized form in figure 86 , would be $\pi D^{3} / 8$, and the volume of the small connecting channels can be called $k \pi D^{3} / 8$, so that the number $n$ of pores in a unit volume of rock having a porosity, $\phi$, is

$$
n=\frac{8 \phi}{(1+k) \pi D^{3}}
$$

If the connecting channels of only a fraction, $N$, of these pore structures are blocked by selectively permeable membranes, the charge stored in a unit volume will be

$$
Q=n N q
$$

Under the application of a unit voltage, the capacity of this unit volume will be equal to the charge, $Q$. Ordinarily, most of the resistance of a rock is contributed by the small pores because of their small cross section and great length. The larger, central pores will have a smaller voltage gradient, smaller by a factor, $m$, because of their smaller resistance per unit length. 
Combining all of these considerations, it is possible to estimate the dielectric constant of a typical, water-saturated sandstone

where

$$
\epsilon=\frac{C}{C_{0}}=\frac{Q}{8.85 \times 10^{-14}}
$$

$C_{0}=$ the capacity of a cubic centimeter of a vacuum, or $8.85 \times 10^{-14}$ farads

Using equations 14 through 18 to replace $Q$, we obtain

$$
\epsilon=\frac{9 \times 10^{13}}{(1+k) \pi D^{5}}(N) \frac{\mu^{2} I t\left(\alpha-\alpha_{0}\right)}{\left(\mu_{+}+\mu_{-}\right)\left(2 \alpha-\alpha_{0}\right)} \int_{t=0}^{t=t_{1}} V_{1}(t) d t
$$

It is necessary to know how the diffusion potential caused by the concentration of anions depends on charging time and pore diameter to evaluate the integral precisely. However, in a qualitative approach, the integral may be evaluated as

$$
\int_{t_{0}}^{t_{1}} V_{1}(t) d t=\frac{A(t, D) t E}{m}
$$

where

$E=$ the externally applied voltage gradient, taken as 1 volt per centimeter for the purposes of this discussion

$A=\mathrm{a}$ constant depending on $t$ and $D$

Also, Archie's law (Archie, 1947) may be used to express the current, $I$, in equation 20 in terms of porosity and water resistivity

where

$$
I=\frac{E \phi^{2} S^{2}}{\rho_{\omega}}
$$

$\rho_{\omega}=$ the resistivity of the water in the pores

$S=$ the fraction of the pores filled with water

Assuming that the pores are fully saturated, equation 20 becomes

$$
\epsilon=9 \times 10^{13} \frac{\phi^{3} A N t^{2} \mu_{-}^{2}\left(a-a_{0}\right)}{(1+k) \pi \bar{D}^{5} \rho_{\omega} m\left(\mu_{+}+\mu_{-}\right)\left(2 a-a_{0}\right)}
$$

\begin{tabular}{|c|c|c|c|}
\hline Parameter & Case 1 & Case 2 & Case 3 \\
\hline 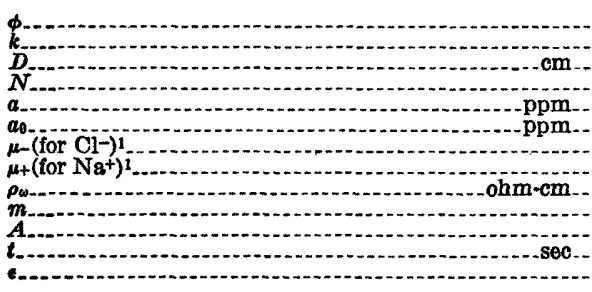 & $\begin{array}{r}0.10 \\
100 \\
.01 \\
50,1 \\
40,000 \\
67.6 \times 10^{-6} \\
45.6 \times 10^{-5} \\
12 \\
20 \\
.1 \\
1 \\
8.05 \times 10^{9}\end{array}$ & $\begin{array}{r}0.16 \\
2 \\
.05 \\
.01 \\
20,000 \\
1,000 \\
67.6 \times 10^{-5} \\
45.6 \times 10^{-5} \\
34 \\
50 \\
.8 \\
1 \\
1.19 \times 10^{8}\end{array}$ & $\begin{array}{r}0.24 \\
.2 \\
2.001 \\
10,000 \\
100 \\
67.6 \times 10^{-8} \\
45.6 \times 10^{-6} \\
65 \\
100 \\
1.0 \\
1.2 \times 10^{4}\end{array}$ \\
\hline
\end{tabular}

An evaluation of this expression for several hypothetical sets of conditions is presented in table 2.

TABLE 2.-Evaluation of dielectric constant under certain hypothetical conditions

1 Values expressed as $\frac{\mathrm{cm} / \mathrm{sec}}{\text { volt/cm }}$. 
The influence of any single factor on the dielectric constant cannot readily be evaluated because of the qualitative nature of this analysis. However, if values which are geologically probable are assumed for the various parameters, it is obvious that the exist ence of a membrane effect would lead to high values of static dielectric constant.

One may infer from such considerations that siltstones will probably have larger dielectric constants than any other rock types. In shales all the negative charges may be immobile, so that no polarization can take place; and in clean sandstones, there may be no anion traps to cause polarization. Siltstones will provide a large number of anion traps and at the same time have a sufficient number of mobile anions to make use of these traps.

Other mechanisms for the origin of large polarizations have been suggested (Dakhnov and others, 1952). In a metal-bearing rock, electrode polarization may take place at every interface between metal and electrolytic solution. The sandstones of the Morrison formation contain only small percentages of metallic minerals, so this mechanism is not of interest. A second phenomenon suggested by Dakhnov is the storage of charge by electroosmosis. When a voltage is applied across a rock, the water in the pores may be carried along with the electric current. Following the cessation of current the displaced water will return to its original position, giving rise to an electrokinetic potential. As the water in sandstones of the Morrison formation is highly saline, it is not likely that an appreciable electrokinetic potential could be generated or that an appreciable charge could be stored.

\section{SUMMARY AND CONCLUSIONS}

The experiments described in this report suggest that the electrical characteristics and probably the nature of electrical conduction in earth materials is considerably more complicated than is commonly assumed in geophysical prospecting. Measurements on water-bearing sandstones and siltstones from the Morrison formation showed a strong dispersion of both resistivity and dielectric constant with frequency. The variation in resistivity is most pronounced in rocks with low water contents; and large dielectric constants are associated with rocks having the higher water contents, such as siltstones and mudstones. If a rock is considered to contain selectively permeable membranes, as has been suggested to explain natural potentials observed in electric logging, it is possible to explain extremely high dielectric constants.

These measurements were made on parts of only 27 cores from 2 neighboring drill holes in a small area of the Colorado Plateau, but there is no reason to believe that these specimens are unique. The high dielectric constants observed with the water-bearing mudstones 
(as great as $10^{6}$ ) might also be observed with any equally wet mudstione or shale. The dispersion of resistivity observed on the sandstones may not be so common, as it appears only with water contents of less than 3 percent of the rock volume; but there are examples of sandstones in desert areas and in oil-wet oil reservoirs where such low saturations are the rule rather than the exception. For this reason the consideration of these phenomena in electrical prospecting should be of general interest to geophysicists.

\section{LITERATURE CITED}

Archie, G. E., 1947, Electrical resistivity-an aid in core analysis interpretation: Am. Assoc. Petroleum Geologists Bull., v. 31, no. 2, p. 350-366.

Dakhnov, V. N., Latyshova, M. G., and Ryapolov, V. A., 1952, Induced polarization well logging: Promyslovaya geofizika, p. 46-82.

Davidson, D. W., and Cole, R. H., 1950, Dielectric relaxation in glycerine: Jour. Chem. Physics, v. 18, no. 10, p. 1417.

Dunlap, W. C., and Makower, B., 1945, Radio frequency dielectric properties of dehydrated carrots: Jour. Phys. Chemistry, v. 49, no. 6, p. 601-621.

Evjen, H. M., 1948, Theory and practice of low-frequency electromagnetic exploration: Geophysics, v. 13, no. 4, p. 584-594.

Fricke, Hugo, 1925, A mathematical treatment of the electrical conductivity and capacity of disperse systems, II: The capacity of a suspension of conducting spheroids surrounded by a nonconducting membrane for a current of low frequency: Phys. Rev., v. 26, no. 6, p. 678-681.

- 1926, The electric capacity of suspensions with special reference to blood: Jour. Gen. Physiology, v. 9, p. 137-152.

Fricke, Hugo, and Curtis, H. S., 1935, The dielectric constant and resistance of colloidal solutions: Phys. Rev., v. 47, no. 12, p. 974-975.

Fuoss, R. M., and Kirkwood, J. C., 1941, Electrical properties of solids, VIII: Dipole moments in polyvinyl chloride-diphenyl systems: Am. Chem. Soc. Jour., v. 63, no. 2, p. 385-394.

Hartshorn, L., 1926, A critical resume of recent work on dielectrics: Inst. Elec. - Engineers Jour., v. 64, p. 1152-1190.

Jaffe, George, and Rider, J. A., 1952, Polarization in electrolytic solutions; Part II-Measurements: Jour. Chem. Physics, v. 20, no. 7, p. 1077-1087.

Jolliffe, C. B., 1923, A study of polarization capacity and resistance at radio frequencies: Phys. Rev., v. 22, no. 3, p. 293-302.

Keller, G. V., 1959, Electrical properties of sandstones of the Morrison formation: U.S. Geol. Survey Bull. 1052-J.

Koops, G. C., 1951, On the dispersion of resistivity and dielectric constant of some semi-conductors at audiofrequencies: Phys. Rev., v. 83, No. 1, p. 121124.

MoCardell, W. M., and Winsauer, W. O., 1953, The origin of electric potentials observed in wells: Petroleum Technology, Am. Inst. Min. Met. Eng. Trans., v. 198, p. $41-50$.

MacDonald, J. R., 1952, Dielectric dispersion in materials having a distribution of relaxation times: Jour. Chem. Physics, v. 20, no. 7, p. 1107-1111.

Manger, G. E., Gates, G. L., and Cadigan, R. A., 1954, Progress report-Original state core studies: Am. Geophys. Union Trans., v. 35, p. 381-382. 
Maxwell, J. C., 1892, Theory of a composite dielectric, in A treatise on electricity and magnetism, p. 452-461.

Smith-Rose, R. L., 1934, Electrical measurements on soil with alternating currents: Inst. Elec. Engineers Jour., v. 75, p. 221-237.

Sollner, Karl, 1945, The physical chemistry of membranes with particular reference to the electrical behavior of membranes of porous character: Jour. Phys. Chemistry, v. 49, p. 47-66, 171-191, 265-280.

Tarkhov, A. G., 1948, Resistivity and dielectric constant of rocks in alternating current fields: Vses. Nauk-Ozled. Geol. Inst., Mater. Geofiz, no. 12, p. 3-42.

Von Hippel, A. R., 1954, Interfacial and space charge polarization: in Dielectrics and Waves, New York, John Wiley and Sons, p. 228-234.

Wagner, K. W., 1913, Zur Theorie der Unvolkommenen Dielektrik: Annalen Physik, Band 40, p. 817-855.

Yager, W. A., 1936, The distribution of relaxation times in typical dielectrics: Physics, v. 7, p. 434-450. 


